Supporting Information

\title{
Harnessing Non-covalent Interactions for a Directed Evolution of a Six-Component Molecular Crystal
}

Sucharita Mandal, Arun K. Pal, Nilangshu Mandal, Titas Kumar Mukhopadhyay, Ayan Datta*

School of Chemical Sciences, Indian Association for the Cultivation of Science, Jadavpur 700032, West Bengal, India.

Corresponding Author: spad@iacs.res.in. Phone: +91-33-24734971. 


\section{Contents:}

1. Cartesian coordinates and energies for DFT optimized molecules.

2. M06-2X/6-31G(d)/ECP optimized structures of the 1:1:1:1:1:1 molecular complexes.

3. Range-separation DFT parameters for the 6-component molecular complex and its individual components.

4. Optimized geometries and cell parameters from Periodic DFT calculations using Crystal17. 


\section{Cartesian coordinates and energies for DFT optimized molecules:}

Crownether(I) at M06-2X/6-31G(d)

$\mathrm{E}=-922.5744003 \mathrm{au}$

No. of imaginary freq. $=0$

\begin{tabular}{|c|c|c|c|}
\hline \multirow[b]{2}{*}{ Atoms } & \multicolumn{2}{|c|}{ Coordinates (Angstroms) } & \multirow[b]{2}{*}{$\mathrm{Z}$} \\
\hline & $X$ & $\mathrm{Y}$ & \\
\hline $\mathrm{C}$ & 3.83204000 & 0.09711100 & 0.18640600 \\
\hline $\mathrm{H}$ & 3.68752900 & -0.10666200 & 1.25977900 \\
\hline $\mathrm{H}$ & 4.84609600 & 0.51643300 & 0.07199700 \\
\hline $\mathrm{C}$ & 2.67346800 & 2.08952100 & 0.58305400 \\
\hline $\mathrm{H}$ & 3.59585000 & 2.68969400 & 0.65478300 \\
\hline $\mathrm{H}$ & 2.40668500 & 1.74788900 & 1.59586200 \\
\hline $\mathrm{C}$ & 1.55646000 & 2.94163700 & 0.02924000 \\
\hline $\mathrm{H}$ & 1.48268300 & 3.87848500 & 0.60645200 \\
\hline $\mathrm{H}$ & 1.79019300 & 3.20334600 & -1.01537000 \\
\hline $\mathrm{C}$ & -0.69543100 & 2.84443300 & -0.57048500 \\
\hline $\mathrm{H}$ & -0.39039700 & 3.10854200 & -1.59681900 \\
\hline $\mathrm{H}$ & -0.98960200 & 3.77167000 & -0.05272000 \\
\hline $\mathrm{C}$ & -1.87767300 & 1.90679900 & -0.64747100 \\
\hline $\mathrm{H}$ & -2.60809900 & 2.32665500 & -1.36082200 \\
\hline $\mathrm{H}$ & -1.53312300 & 0.93248200 & -1.02193600 \\
\hline $\mathrm{C}$ & -3.73425400 & 1.19087700 & 0.61626600 \\
\hline $\mathrm{H}$ & -4.47986300 & 1.89816900 & 0.21247800 \\
\hline $\mathrm{H}$ & -3.97934100 & 0.98993300 & 1.66260300 \\
\hline
\end{tabular}




\begin{tabular}{|c|c|c|c|}
\hline $\mathrm{O}$ & 2.85414600 & 0.98978700 & -0.27243800 \\
\hline $\mathrm{O}$ & 0.36290100 & 2.20953500 & 0.09827200 \\
\hline $\mathrm{O}$ & -2.45455200 & 1.77168500 & 0.62833500 \\
\hline $\mathrm{C}$ & -3.83203800 & -0.09710700 & -0.18640500 \\
\hline $\mathrm{H}$ & -3.68752300 & 0.10667100 & -1.25977700 \\
\hline $\mathrm{H}$ & -4.84609500 & -0.51642800 & -0.07200100 \\
\hline $\mathrm{C}$ & -2.67346700 & -2.08951600 & -0.58305900 \\
\hline $\mathrm{H}$ & -3.59584900 & -2.68968800 & -0.65479300 \\
\hline $\mathrm{H}$ & -2.40668100 & -1.74788000 & -1.59586600 \\
\hline $\mathrm{C}$ & -1.55646100 & -2.94163600 & -0.02924700 \\
\hline $\mathrm{H}$ & -1.48268400 & -3.87848200 & -0.60646300 \\
\hline $\mathrm{H}$ & -1.79019700 & -3.20335000 & 1.01536100 \\
\hline $\mathrm{C}$ & 0.69542900 & -2.84443700 & 0.57048400 \\
\hline $\mathrm{H}$ & 0.39039200 & -3.10855000 & 1.59681600 \\
\hline $\mathrm{H}$ & 0.98960100 & -3.77167300 & 0.05271600 \\
\hline $\mathrm{C}$ & 1.87767100 & -1.90680400 & 0.64747500 \\
\hline $\mathrm{H}$ & 2.60809400 & -2.32666200 & 1.36082700 \\
\hline $\mathrm{H}$ & 1.53312000 & -0.93248700 & 1.02194200 \\
\hline $\mathrm{C}$ & 3.73425600 & -1.19087600 & -0.61625900 \\
\hline $\mathrm{H}$ & 4.47986600 & -1.89816700 & -0.21246900 \\
\hline $\mathrm{H}$ & 3.97934300 & -0.98993600 & -1.66259700 \\
\hline $\mathrm{O}$ & -2.85414500 & -0.98978700 & 0.27243700 \\
\hline $\mathrm{O}$ & -0.36290100 & -2.20953600 & -0.09827400 \\
\hline $\mathrm{O}$ & 2.45455400 & -1.77168600 & -0.62832900 \\
\hline
\end{tabular}


Thiourea (II) at M06-2X/6-31G(d)

$E=-548.1086159 a u$

No. of imaginary freq. $=0$

Coordinates (Angstroms)

$\begin{array}{llll}\text { Atoms } & \mathrm{X} & \mathrm{Y} & \mathrm{Z}\end{array}$

C -0.3133820

$\mathrm{N}$

$-1.05107000$

$-0.00000100$

0.00002800

$\mathrm{H}$

$-1.99815300$

1.14202400

$-0.05680700$

$\mathrm{H}$

$$
-0.53159500
$$

1.14233500

0.29902700

$\mathrm{N}$

$-1.05106800$

2.00037700

0.05622000

$\mathrm{H}$

$-1.14202600$

0.05679500

$\begin{array}{ll}\mathrm{H} & -1.99815400\end{array}$

$-1.14232500$

$-0.29903400$

$\mathrm{H}$

$-0.53159300$

$-2.00037800$

$-0.05624600$

S

1.35342200

0.00000100

$-0.00000300$

2,3,5,6-tetrafluro-1,4-dibromobenzene (III) at M06-2X/6-31G(d)

$\mathrm{E}=-653.9533988 \mathrm{au}$

No. of imaginary freq. $=0$

Coordinates (Angstroms)

$\begin{array}{llll}\text { Atoms } & \mathrm{X} & \mathrm{Y} & \mathrm{Z}\end{array}$

$\begin{array}{llll}\text { C } & 1.39626800 & -0.00009200 & -0.00049500 \\ \text { C } & 0.69500800 & -1.19863400 & -0.00037400 \\ \text { C } & -0.69497400 & -1.19865700 & 0.00017200 \\ \text { C } & -1.39626700 & -0.00017000 & 0.00035800\end{array}$




$\begin{array}{llll}\mathrm{C} & -0.69503900 & 1.19846600 & 0.00019500 \\ \mathrm{C} & 0.69493500 & 1.19846300 & -0.00040900 \\ \mathrm{Br} & 3.31062600 & 0.00012800 & 0.00020600 \\ \mathrm{Br} & -3.31060900 & 0.00010300 & -0.00006000 \\ \mathrm{~F} & 1.33153500 & -2.36148700 & -0.00039600 \\ \mathrm{~F} & -1.33145600 & -2.36153400 & 0.00029300 \\ \mathrm{~F} & -1.33159100 & 2.36124600 & 0.00039300 \\ \mathrm{~F} & 1.33149400 & 2.36129200 & -0.00049300\end{array}$

Ammonium-cation (IV) at M06-2X/6-31G(d)

$\mathrm{E}=-56.8556724 \mathrm{au}$

No. of imaginary freq. $=0$

Coordinates (Angstroms)

$\begin{array}{llll}\text { Atoms } & \mathrm{X} & \mathrm{Y} & \mathrm{Z}\end{array}$

Perchlorate-anion (V) at M06-2X/6-31G(d)

$\mathrm{E}=-760.6510688 \mathrm{au}$

No. of imaginary freq. $=0$

Coordinates (Angstroms) 
$\begin{array}{llll}\text { Atoms } & \mathrm{X} & \mathrm{Y} & \mathrm{Z}\end{array}$

$\begin{array}{llll}\mathrm{Cl} & 0.00000000 & 0.00000000 & 0.00000000 \\ \mathrm{O} & -0.85331700 & -0.85331700 & 0.85331700 \\ \mathrm{O} & 0.85331700 & 0.85331700 & 0.85331700 \\ \mathrm{O} & -0.85331700 & 0.85331700 & -0.85331700 \\ \mathrm{O} & 0.85331700 & -0.85331700 & -0.85331700\end{array}$

Bisulfate-anion (V) at M06-2X/6-31G(d)

$E=-699.3759205 a u$

No. of imaginary freq. $=0$

Coordinates (Angstroms)

$\begin{array}{llll}\text { Atoms } & \mathrm{X} & \mathrm{Y} & \mathrm{Z}\end{array}$

$\begin{array}{llll}\mathrm{S} & -2.73842800 & 2.57931000 & 0.96570700 \\ \mathrm{O} & -3.97160800 & 1.72955200 & 1.51197100 \\ \mathrm{O} & -1.55187800 & 1.68223400 & 1.02432900 \\ \mathrm{O} & -2.88108200 & 2.62285200 & -0.51556800 \\ \mathrm{O} & -2.71116600 & 3.81553800 & 1.69644600 \\ \mathrm{H} & -4.41711100 & 2.24549800 & 2.20825900\end{array}$

Bicarbonate-anion (V) at M06-2X/6-31G(d)

$E=-264.2124344 a u$

No. of imaginary freq. $=0$

Coordinates (Angstroms) 
$\begin{array}{llll}\text { Atoms } & \mathrm{X} & \mathrm{Y} & \mathrm{Z}\end{array}$

$\begin{array}{llll}\mathrm{C} & -2.49997600 & 1.39939300 & 1.15256200 \\ \mathrm{O} & -2.01774600 & 2.11642300 & 0.17901200 \\ \mathrm{O} & -1.95841300 & 0.34860900 & 1.41639800 \\ \mathrm{O} & -3.55849400 & 1.92999900 & 1.75023100 \\ \mathrm{H} & -3.83317000 & 1.31598600 & 2.45344400\end{array}$

Hexamethylbenzeneenzene (VI) at M06-2X/6-31G(d)

$E=-467.9057338 a u$

No. of imaginary freq. $=0$

Coordinates (Angstroms)

$\begin{array}{llll}\text { Atoms } & \mathrm{X} & \mathrm{Y} & \mathrm{Z}\end{array}$

$\begin{array}{llll}\mathrm{C} & -1.39862800 & 0.10613500 & 0.01112100 \\ \mathrm{C} & -0.60740800 & 1.26432600 & -0.01105100 \\ \mathrm{C} & 0.79118600 & 1.15820100 & 0.01099200 \\ \mathrm{C} & 1.39861400 & -0.10613600 & -0.01107400 \\ \mathrm{C} & 0.60740800 & -1.26431400 & 0.01105700 \\ \mathrm{C} & -0.79121000 & -1.15818800 & -0.01100900 \\ \mathrm{H} & -1.64447300 & -2.40663100 & -0.08622000 \\ \mathrm{H} & -1.90804600 & -2.78938800 & -0.61865500 \\ \mathrm{H} & -2.57742800 & -2.21819700 & 0.90860200 \\ \mathrm{C} & 1.26223900 & -2.62735200 & -0.62129400 \\ \mathrm{H} & 0.63216200 & -3.34177900 & 0.08609800 \\ \mathrm{H} & 1.46351400 & -3.04609100 & -0.9087976000 \\ & & \mathrm{~S} & \end{array}$




$\begin{array}{llll}\mathrm{H} & 2.21364900 & -2.58205400 & 0.61991200 \\ \mathrm{C} & 2.90646000 & -0.22067700 & -0.08601800 \\ \mathrm{H} & 3.36951700 & -0.25729600 & 0.90891700 \\ \mathrm{H} & 3.21003100 & -1.12292700 & -0.62083000 \\ \mathrm{H} & 3.34325700 & 0.62656000 & -0.61852200 \\ \mathrm{C} & 1.64443300 & 2.40666500 & 0.08602300 \\ \mathrm{H} & 2.57716900 & 2.21847100 & 0.62156600 \\ \mathrm{H} & 1.90840700 & 2.78896900 & -0.90886600 \\ \mathrm{H} & 1.12881200 & 3.20879900 & 0.61788100 \\ \mathrm{C} & -1.26217600 & 2.62739300 & -0.08609400 \\ \mathrm{H} & -1.46269900 & 3.04648800 & 0.90878800 \\ \mathrm{H} & -0.63234100 & 3.34157200 & -0.62039600 \\ \mathrm{H} & -2.21392500 & 2.58207500 & -0.61928000 \\ \mathrm{C} & -2.90647400 & 0.22058800 & 0.08619000 \\ \mathrm{H} & -3.21007900 & 1.12294500 & 0.62079300 \\ \mathrm{H} & -3.36967400 & 0.25685900 & -0.90869000 \\ \mathrm{H} & -3.34309700 & -0.62655200 & 0.61900300\end{array}$

I-II at M06-2X/6-31G(d)

$\mathrm{E}=-1470.719947985896 \mathrm{au} \quad \mathrm{E}_{\mathrm{bsse}}=-1470.719947958896 \mathrm{au}$

No. of imaginary freq. $=0$

Coordinates (Angstroms)

$\begin{array}{llll}\text { Atoms } & \mathrm{X} & \mathrm{Y} & \mathrm{Z}\end{array}$

$\begin{array}{llll}\mathrm{C} & 0.07235700 & -0.22502800 & 1.73666800 \\ \mathrm{~N} & 0.95021500 & 0.62540400 & 2.34893000 \\ \mathrm{H} & 1.64690900 & 1.05053500 & 1.74235200\end{array}$




\begin{tabular}{|c|c|c|c|}
\hline $\mathrm{H}$ & 1.26957300 & 0.31193600 & 3.25412300 \\
\hline $\mathrm{N}$ & -0.23145500 & 0.08911300 & 0.47769100 \\
\hline $\mathrm{H}$ & 0.25411400 & 0.84749500 & 0.00866000 \\
\hline $\mathrm{H}$ & -0.66634800 & -0.62144700 & -0.10544000 \\
\hline$S$ & -0.63886600 & -1.51676000 & 2.57105000 \\
\hline $\mathrm{C}$ & 2.85543700 & -1.97205100 & -0.77648000 \\
\hline $\mathrm{H}$ & 2.98454600 & -1.66664000 & -1.82870400 \\
\hline $\mathrm{H}$ & 3.39230400 & -2.92846400 & -0.65437000 \\
\hline $\mathrm{C}$ & 0.85798600 & -3.09825300 & -1.23743900 \\
\hline $\mathrm{H}$ & 1.40283100 & -4.05434200 & -1.17181800 \\
\hline $\mathrm{H}$ & 0.82718400 & -2.79342600 & -2.29550300 \\
\hline $\mathrm{C}$ & -0.54786100 & -3.30391400 & -0.72055500 \\
\hline $\mathrm{H}$ & -0.99729900 & -4.15839400 & -1.25054500 \\
\hline $\mathrm{H}$ & -0.52051200 & -3.51473300 & 0.35709100 \\
\hline $\mathrm{C}$ & -2.66341700 & -2.24918400 & -0.61573100 \\
\hline $\mathrm{H}$ & -2.78791800 & -2.93625000 & 0.23226500 \\
\hline $\mathrm{H}$ & -3.22192000 & -2.63735500 & -1.47931200 \\
\hline $\mathrm{C}$ & -3.19272200 & -0.89149700 & -0.19726000 \\
\hline $\mathrm{H}$ & -4.28340600 & -0.96609800 & -0.05112200 \\
\hline $\mathrm{H}$ & -2.73375400 & -0.61283900 & 0.76082600 \\
\hline $\mathrm{C}$ & -3.42709700 & 1.32246400 & -0.96641800 \\
\hline $\mathrm{H}$ & -4.47560400 & 1.23853900 & -0.63715300 \\
\hline $\mathrm{H}$ & -3.40900300 & 1.85377500 & -1.92289400 \\
\hline $\mathrm{O}$ & 1.49994300 & -2.11552800 & -0.46002000 \\
\hline $\mathrm{O}$ & -1.29657200 & -2.13275900 & -0.96194700 \\
\hline $\mathrm{O}$ & -2.89300700 & 0.04320700 & -1.21052400 \\
\hline $\mathrm{C}$ & -2.66207000 & 2.14860800 & 0.05574700 \\
\hline $\mathrm{H}$ & -2.44121000 & 1.55558100 & 0.95568000 \\
\hline $\mathrm{H}$ & -3.28749700 & 3.00640200 & 0.35608000 \\
\hline
\end{tabular}




$\begin{array}{llll}\mathrm{C} & -0.70212400 & 3.37456500 & 0.38124200 \\ \mathrm{H} & -1.20371600 & 4.32944200 & 0.60833900 \\ \mathrm{H} & -0.55622700 & 2.82594100 & 1.32590600 \\ \mathrm{C} & 0.65078300 & 3.63966700 & -0.24313500 \\ \mathrm{H} & 1.21721500 & 4.36217900 & 0.36279200 \\ \mathrm{H} & 0.52390500 & 4.04841800 & -1.25604600 \\ \mathrm{C} & 2.56792000 & 2.39471300 & -0.95204900 \\ \mathrm{H} & 2.49942800 & 2.89504400 & -1.92944200 \\ \mathrm{H} & 3.32589600 & 2.91074400 & -0.34436000 \\ \mathrm{C} & 2.94153800 & 0.93931100 & -1.14674700 \\ \mathrm{H} & 3.93027300 & 0.86534700 & -1.62751600 \\ \mathrm{H} & 2.19501400 & 0.46293200 & -1.79781700 \\ \mathrm{C} & 3.48900700 & -0.96717900 & 0.17074800 \\ \mathrm{H} & 4.57089100 & -0.93035800 & -0.03681100 \\ \mathrm{H} & 3.33502700 & -1.30729500 & 1.19841300 \\ \mathrm{O} & -1.46121900 & 2.61174900 & -0.52311700 \\ \mathrm{O} & 1.32464400 & 2.40236100 & -0.29680400 \\ \mathrm{O} & 2.94638900 & 0.33553600 & 0.12386600\end{array}$

I-II-III at M06-2X/6-31G(d)

$\mathrm{E}=-2124.6884427 \mathrm{au} \quad \mathrm{E}_{\mathrm{bsse}}=-2124.672757009063 \mathrm{au}$

No. of imaginary freq. $=0$

Coordinates (Angstroms)

$\begin{array}{llll}\text { Atoms } & \mathrm{X} & \mathrm{Y} & \mathrm{Z}\end{array}$

$\begin{array}{llll}\mathrm{C} & 2.56791200 & 0.31000000 & 1.28694100 \\ \mathrm{~N} & 3.38303400 & 0.09354500 & 0.26586400\end{array}$




\begin{tabular}{|c|c|c|c|}
\hline $\mathrm{H}$ & 3.60769400 & 0.85374700 & -0.36917100 \\
\hline $\mathrm{H}$ & 3.52140500 & -0.85632000 & -0.06606500 \\
\hline $\mathrm{N}$ & 2.27953500 & 1.62230900 & 1.54354000 \\
\hline $\mathrm{H}$ & 2.92801300 & 2.31402100 & 1.17896300 \\
\hline $\mathrm{H}$ & 1.87264900 & 1.79817400 & 2.45055700 \\
\hline S & 1.91407500 & -0.92865900 & 2.24824600 \\
\hline $\mathrm{C}$ & 6.61139500 & -1.70340600 & 0.09574200 \\
\hline $\mathrm{H}$ & 6.97411900 & -1.57918300 & -0.93629400 \\
\hline $\mathrm{H}$ & 7.24123900 & -2.45950500 & 0.59205800 \\
\hline $\mathrm{C}$ & 6.72686900 & -0.39307800 & 0.84274900 \\
\hline $\mathrm{H}$ & 6.10344800 & -0.45562600 & 1.74714500 \\
\hline $\mathrm{H}$ & 7.77308100 & -0.22801400 & 1.14806300 \\
\hline $\mathrm{C}$ & 6.26088900 & 1.87832200 & 0.73090700 \\
\hline $\mathrm{H}$ & 7.26731800 & 2.14010000 & 1.09768000 \\
\hline $\mathrm{H}$ & 5.59228800 & 1.79721800 & 1.60360600 \\
\hline $\mathrm{C}$ & 5.76059400 & 2.97708000 & -0.17928700 \\
\hline $\mathrm{H}$ & 6.02447100 & 3.96205600 & 0.23439500 \\
\hline $\mathrm{H}$ & 6.23625900 & 2.87413600 & -1.16462400 \\
\hline $\mathrm{C}$ & 3.81253100 & 3.70769800 & -1.29637700 \\
\hline $\mathrm{H}$ & 4.22354000 & 3.43707600 & -2.27913500 \\
\hline $\mathrm{H}$ & 4.05744800 & 4.76107700 & -1.09125800 \\
\hline $\mathrm{C}$ & 2.31410200 & 3.51381800 & -1.29394300 \\
\hline $\mathrm{H}$ & 1.91281100 & 3.73868100 & -0.29299200 \\
\hline $\mathrm{H}$ & 1.84902400 & 4.21408500 & -2.00688500 \\
\hline $\mathrm{O}$ & 5.26786800 & -2.10406800 & 0.11788800 \\
\hline $\mathrm{O}$ & 6.29704400 & 0.66860200 & 0.02098400 \\
\hline $\mathrm{O}$ & 4.35720500 & 2.87025000 & -0.29926900 \\
\hline $\mathrm{C}$ & 0.68728500 & 1.84849400 & -1.46316700 \\
\hline $\mathrm{H}$ & 0.45669200 & 1.81054600 & -0.38916500 \\
\hline
\end{tabular}




\begin{tabular}{|c|c|c|c|}
\hline $\mathrm{H}$ & 0.04588100 & 2.60641300 & -1.94173600 \\
\hline $\mathrm{C}$ & 0.40592600 & 0.50752000 & -2.09692200 \\
\hline $\mathrm{H}$ & 0.71384400 & 0.52309100 & -3.15508400 \\
\hline $\mathrm{H}$ & -0.68042600 & 0.32355000 & -2.05950900 \\
\hline $\mathrm{C}$ & 0.77750000 & -1.77970000 & -1.84227600 \\
\hline $\mathrm{H}$ & -0.31136400 & -1.94229400 & -1.80193000 \\
\hline $\mathrm{H}$ & 1.11101000 & -1.91800700 & -2.88335800 \\
\hline $\mathrm{C}$ & 1.45202500 & -2.78743800 & -0.94404700 \\
\hline $\mathrm{H}$ & 1.12576900 & -3.79985800 & -1.23374500 \\
\hline $\mathrm{H}$ & 1.16782900 & -2.60217600 & 0.10081600 \\
\hline $\mathrm{C}$ & 3.54481000 & -3.62802900 & -0.32662000 \\
\hline $\mathrm{H}$ & 3.26222000 & -3.54477300 & 0.73308500 \\
\hline $\mathrm{H}$ & 3.31421200 & -4.64648300 & -0.67907600 \\
\hline $\mathrm{C}$ & 5.02324800 & -3.35250900 & -0.47596400 \\
\hline $\mathrm{H}$ & 5.29778900 & -3.33851600 & -1.54200100 \\
\hline $\mathrm{H}$ & 5.60630200 & -4.14287900 & 0.02359000 \\
\hline $\mathrm{O}$ & 2.04830300 & 2.18411800 & -1.64831900 \\
\hline $\mathrm{O}$ & 1.09415400 & -0.48724300 & -1.39214300 \\
\hline $\mathrm{O}$ & 2.85216900 & -2.67109300 & -1.09277500 \\
\hline $\mathrm{C}$ & -3.00994900 & -0.25934900 & 0.60730900 \\
\hline $\mathrm{C}$ & -3.48809100 & -0.60738900 & -0.64817700 \\
\hline $\mathrm{C}$ & -4.82736800 & -0.44163800 & -0.97820800 \\
\hline $\mathrm{C}$ & -5.71522700 & 0.07587100 & -0.04489700 \\
\hline $\mathrm{C}$ & -5.24746800 & 0.42478300 & 1.21480000 \\
\hline $\mathrm{C}$ & -3.90528600 & 0.25678600 & 1.53525400 \\
\hline $\mathrm{Br}$ & -1.15277000 & -0.49312300 & 1.07183200 \\
\hline $\mathrm{Br}$ & -7.56434600 & 0.30735300 & -0.49032900 \\
\hline $\mathrm{F}$ & -2.67234000 & -1.10494300 & -1.57780200 \\
\hline $\mathrm{F}$ & -5.23676400 & -0.78517500 & -2.19344400 \\
\hline
\end{tabular}




$\begin{array}{llll}\text { F } & -6.06747700 & 0.92270700 & 2.13160900 \\ \text { F } & -3.50150900 & 0.60492500 & 2.75051300\end{array}$

I-II-III-IV at M06-2X/6-31G(d)
$\mathrm{E}=-2181.6252821 \mathrm{au}$
$E_{b s s e}=-2181.605125452695 a u$

No. of imaginary freq. $=0$

Coordinates (Angstroms)

$\begin{array}{llll}\text { Atoms } & \mathrm{X} & \mathrm{Y} & \mathrm{Z}\end{array}$

\begin{tabular}{|c|c|c|c|}
\hline $\mathrm{C}$ & -2.72954300 & 0.34796900 & 1.44475000 \\
\hline $\mathrm{N}$ & -2.92459800 & 1.59191400 & 1.97729600 \\
\hline $\mathrm{H}$ & -3.73710100 & 2.11994000 & 1.66554100 \\
\hline $\mathrm{H}$ & -2.64743700 & 1.69130600 & 2.94471100 \\
\hline $\mathrm{N}$ & -3.49626800 & -0.01728100 & 0.43787800 \\
\hline $\mathrm{H}$ & -4.36439400 & 0.46879300 & 0.21516200 \\
\hline $\mathrm{H}$ & -3.30342200 & -0.91675700 & -0.01485500 \\
\hline S & -1.44959900 & -0.62386300 & 2.04205200 \\
\hline $\mathrm{C}$ & -1.43884500 & 0.09202000 & -2.78379700 \\
\hline $\mathrm{H}$ & -2.45949700 & 0.05199000 & -3.19469200 \\
\hline $\mathrm{H}$ & -0.74912100 & -0.21805800 & -3.58513800 \\
\hline $\mathrm{C}$ & -1.39819000 & -2.12940100 & -2.05452200 \\
\hline $\mathrm{H}$ & -0.45782100 & -2.43172900 & -2.53884600 \\
\hline $\mathrm{H}$ & -2.22276800 & -2.27565500 & -2.76595300 \\
\hline $\mathrm{C}$ & -1.65899100 & -2.97389300 & -0.83028000 \\
\hline $\mathrm{H}$ & -1.61772800 & -4.03530100 & -1.11395700 \\
\hline
\end{tabular}




\begin{tabular}{|c|c|c|c|}
\hline $\mathrm{H}$ & -0.90994800 & -2.78060700 & -0.04914900 \\
\hline $\mathrm{C}$ & -3.46217200 & -3.51293900 & 0.61738000 \\
\hline $\mathrm{H}$ & -2.69403700 & -3.75391700 & 1.36488900 \\
\hline $\mathrm{H}$ & -3.79769900 & -4.43965900 & 0.13259700 \\
\hline $\mathrm{C}$ & -4.62451000 & -2.82100600 & 1.30609800 \\
\hline $\mathrm{H}$ & -5.15418800 & -3.55109600 & 1.93657900 \\
\hline $\mathrm{H}$ & -4.24388100 & -2.01975700 & 1.95583200 \\
\hline $\mathrm{C}$ & -6.72022300 & -1.84914000 & 0.76837400 \\
\hline $\mathrm{H}$ & -7.13737600 & -2.53787900 & 1.51874100 \\
\hline $\mathrm{H}$ & -7.38690900 & -1.83934800 & -0.09859200 \\
\hline $\mathrm{O}$ & -1.32967800 & -0.76343100 & -1.67128000 \\
\hline $\mathrm{O}$ & -2.94567300 & -2.63797400 & -0.36543200 \\
\hline $\mathrm{O}$ & -5.47587200 & -2.30993100 & 0.30489300 \\
\hline $\mathrm{C}$ & -6.68636300 & -0.44974200 & 1.36031700 \\
\hline $\mathrm{H}$ & -5.96541600 & -0.37551800 & 2.18963000 \\
\hline $\mathrm{H}$ & -7.68482100 & -0.21751200 & 1.75691900 \\
\hline $\mathrm{C}$ & -6.74893700 & 1.80009900 & 0.63468800 \\
\hline $\mathrm{H}$ & -7.83628900 & 1.90291600 & 0.51548200 \\
\hline $\mathrm{H}$ & -6.49003100 & 2.07483800 & 1.66913000 \\
\hline $\mathrm{C}$ & -6.02930300 & 2.72422900 & -0.31874000 \\
\hline $\mathrm{H}$ & -6.44618600 & 3.73840900 & -0.26291300 \\
\hline $\mathrm{H}$ & -6.14239600 & 2.34885400 & -1.34490600 \\
\hline $\mathrm{C}$ & -3.81022600 & 3.43446600 & -0.83693100 \\
\hline $\mathrm{H}$ & -4.37837000 & 4.22212800 & -1.34667600 \\
\hline $\mathrm{H}$ & -3.02944900 & 3.91806900 & -0.24014800 \\
\hline $\mathrm{C}$ & -3.14822400 & 2.52530200 & -1.86637600 \\
\hline
\end{tabular}




\begin{tabular}{|c|c|c|c|}
\hline $\mathrm{H}$ & -2.98224500 & 3.08154300 & -2.79940700 \\
\hline $\mathrm{H}$ & -3.80437800 & 1.67084400 & -2.08162700 \\
\hline $\mathrm{C}$ & -1.06023300 & 1.50373400 & -2.38265400 \\
\hline $\mathrm{H}$ & -1.05711100 & 2.14733300 & -3.27331000 \\
\hline $\mathrm{H}$ & -0.04646700 & 1.49208100 & -1.96996500 \\
\hline $\mathrm{O}$ & -6.34100400 & 0.48135300 & 0.34533900 \\
\hline $\mathrm{O}$ & -4.66002100 & 2.74190600 & 0.05871600 \\
\hline $\mathrm{O}$ & -1.88907200 & 2.07767200 & -1.38145300 \\
\hline $\mathrm{C}$ & 3.29420300 & -0.34660600 & -0.29706600 \\
\hline $\mathrm{C}$ & 3.69362900 & 0.91823300 & 0.10551300 \\
\hline $\mathrm{C}$ & 5.01880900 & 1.18978500 & 0.40916500 \\
\hline $\mathrm{C}$ & 5.97143400 & 0.18204500 & 0.30867500 \\
\hline $\mathrm{C}$ & 5.57662100 & -1.08759200 & -0.09515000 \\
\hline $\mathrm{C}$ & 4.24348200 & -1.35265900 & -0.39651200 \\
\hline $\mathrm{F}$ & 2.79619400 & 1.91559500 & 0.20992900 \\
\hline $\mathrm{F}$ & 5.34900300 & 2.41354900 & 0.79115000 \\
\hline $\mathrm{F}$ & 6.45440900 & -2.06926000 & -0.20215100 \\
\hline $\mathrm{F}$ & 3.90120500 & -2.57278100 & -0.77820500 \\
\hline $\mathrm{Br}$ & 7.80134900 & 0.54159000 & 0.72166500 \\
\hline $\mathrm{Br}$ & 1.44696600 & -0.68417500 & -0.70569600 \\
\hline $\mathrm{N}$ & -0.16446400 & 1.94744000 & 0.87063900 \\
\hline $\mathrm{H}$ & -0.42214700 & 1.02799500 & 1.36338400 \\
\hline $\mathrm{H}$ & -0.86980400 & 2.09243200 & 0.11794900 \\
\hline $\mathrm{H}$ & -0.21762000 & 2.71640800 & 1.54076400 \\
\hline $\mathrm{H}$ & 0.78468500 & 1.87459100 & 0.48999500 \\
\hline
\end{tabular}


I-II-III-IV-V of system A at M06-2X/6-31G(d)

$$
\mathrm{E}=-2942.4180837 \mathrm{au} \quad \mathrm{E}_{\mathrm{bsse}}=-2942.386394760549 \mathrm{au}
$$

No. of imaginary freq. $=0$

Coordinates (Angstroms)

$\begin{array}{llll}\text { Atoms } & \mathrm{X} & \mathrm{Y} & \mathrm{Z}\end{array}$

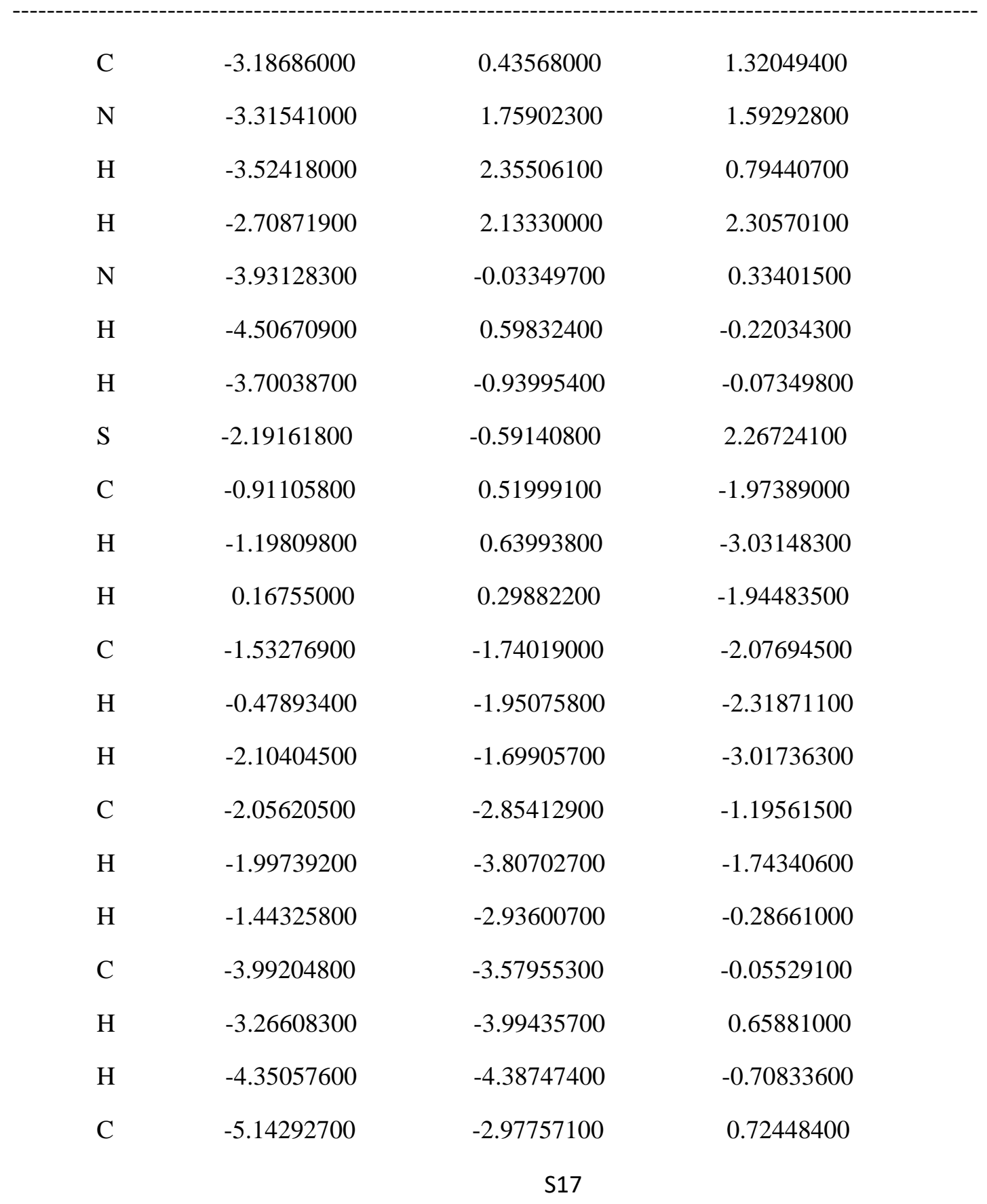




\begin{tabular}{|c|c|c|c|}
\hline $\mathrm{H}$ & -5.64833300 & -3.78025100 & 1.28614400 \\
\hline $\mathrm{H}$ & -4.75028500 & -2.24851800 & 1.44837500 \\
\hline $\mathrm{C}$ & -7.21135300 & -1.89123200 & 0.39732600 \\
\hline $\mathrm{H}$ & -7.58723800 & -2.60146100 & 1.15076100 \\
\hline $\mathrm{H}$ & -7.94976900 & -1.81910200 & -0.40678100 \\
\hline $\mathrm{O}$ & -1.64975600 & -0.52225400 & -1.38232600 \\
\hline $\mathrm{O}$ & -3.39057200 & -2.57077200 & -0.84074700 \\
\hline $\mathrm{O}$ & -6.02876200 & -2.37741400 & -0.18986000 \\
\hline $\mathrm{C}$ & -7.07000400 & -0.52077700 & 1.03819700 \\
\hline $\mathrm{H}$ & -6.21538700 & -0.49132500 & 1.73199700 \\
\hline $\mathrm{H}$ & -7.98517100 & -0.30295500 & 1.61328900 \\
\hline $\mathrm{C}$ & -6.87311100 & 1.75406100 & 0.51073300 \\
\hline $\mathrm{H}$ & -7.86747800 & 2.05808600 & 0.87402700 \\
\hline $\mathrm{H}$ & -6.15801600 & 1.84405200 & 1.34467900 \\
\hline $\mathrm{C}$ & -6.43048900 & 2.65782800 & -0.61967300 \\
\hline $\mathrm{H}$ & -6.51834300 & 3.71444700 & -0.32770600 \\
\hline $\mathrm{H}$ & -7.05769800 & 2.48134300 & -1.50491300 \\
\hline $\mathrm{C}$ & -4.51866900 & 2.94444400 & -2.03009500 \\
\hline $\mathrm{H}$ & -5.19075200 & 2.86203400 & -2.89608900 \\
\hline $\mathrm{H}$ & -4.33396500 & 4.00865700 & -1.82534400 \\
\hline $\mathrm{C}$ & -3.21554600 & 2.22247800 & -2.31246000 \\
\hline $\mathrm{H}$ & -2.68644600 & 2.72241900 & -3.13875900 \\
\hline $\mathrm{H}$ & -3.42813100 & 1.18356400 & -2.60516500 \\
\hline $\mathrm{C}$ & -1.13412600 & 1.80056900 & -1.19338300 \\
\hline $\mathrm{H}$ & -0.48540600 & 2.58318000 & -1.60980300 \\
\hline $\mathrm{H}$ & -0.83408600 & 1.63207600 & -0.15551400 \\
\hline $\mathrm{O}$ & -6.89764400 & 0.43771100 & 0.01857900 \\
\hline $\mathrm{O}$ & -5.08883900 & 2.32618700 & -0.89760800 \\
\hline $\mathrm{O}$ & -2.47483400 & 2.25396100 & -1.12150200 \\
\hline
\end{tabular}




\begin{tabular}{|c|c|c|c|}
\hline $\mathrm{C}$ & 2.70452600 & -1.10169600 & 0.15984500 \\
\hline $\mathrm{C}$ & 3.53941300 & -0.84217700 & 1.23360200 \\
\hline $\mathrm{C}$ & 4.89336400 & -0.61256600 & 1.03991300 \\
\hline $\mathrm{C}$ & 5.42530700 & -0.66298100 & -0.24051900 \\
\hline $\mathrm{C}$ & 4.58855000 & -0.90490900 & -1.32159500 \\
\hline $\mathrm{C}$ & 3.23161000 & -1.11790000 & -1.12164300 \\
\hline $\mathrm{F}$ & 3.04773200 & -0.79784100 & 2.47855500 \\
\hline $\mathrm{F}$ & 5.65507300 & -0.35655800 & 2.09175600 \\
\hline $\mathrm{F}$ & 5.06398700 & -0.94093100 & -2.55889000 \\
\hline $\mathrm{F}$ & 2.44841700 & -1.34197600 & -2.17449600 \\
\hline $\mathrm{Br}$ & 7.28939400 & -0.33439000 & -0.52072800 \\
\hline $\mathrm{Br}$ & 0.83027000 & -1.39880500 & 0.44160300 \\
\hline $\mathrm{O}$ & 3.89970900 & 1.93678000 & -0.44309400 \\
\hline $\mathrm{O}$ & 1.54259300 & 1.88872900 & 0.06325800 \\
\hline $\mathrm{O}$ & 3.13153200 & 1.97592300 & 1.85042300 \\
\hline $\mathrm{O}$ & 2.81573400 & 3.91188500 & 0.43729200 \\
\hline $\mathrm{N}$ & 0.62201900 & 1.07214500 & 2.50390600 \\
\hline $\mathrm{H}$ & -0.31041200 & 0.58798700 & 2.52896200 \\
\hline $\mathrm{H}$ & 0.83889900 & 1.41892300 & 1.53010700 \\
\hline $\mathrm{H}$ & 0.67148700 & 1.86074000 & 3.15117400 \\
\hline $\mathrm{H}$ & 1.38406900 & 0.42299800 & 2.73049000 \\
\hline $\mathrm{Cl}$ & 2.88340500 & 2.45685800 & 0.46792700 \\
\hline
\end{tabular}

\section{I-II-III-IV-V of system B at M06-2X/6-31G(d)}
$\mathrm{E}=-2881.3245353 \mathrm{au}$
$\mathrm{E}_{\mathrm{bsse}}=-2881.291624945445 \mathrm{au}$

No. of imaginary freq. $=0$ 
$\begin{array}{llll}\text { Atoms } & \mathrm{X} & \mathrm{Y} & \mathrm{Z}\end{array}$

C $\quad-3.20819100$

0.43612400

1.33505700

$\mathrm{N}$

$-3.36753700$

1.75580500

1.61700200

$\mathrm{H}$

$-3.57537400$

2.35015800

0.81677200

$\mathrm{H}$

$-2.75708900$

2.13481000

2.32447500

$\mathrm{N}$

$-3.94403200$

$-0.04088400$

0.34472800

$\mathrm{H}$

$-4.52747900$

0.58421300

$-0.20861700$

$\mathrm{H}$

$-3.69196000$

$-0.93756800$

$-0.07123100$

S

$-2.18933200$

$-0.57230400$

2.27301200

C

$-0.91014600$

0.57929100

$-1.92677700$

$\mathrm{H}$

$-1.17739200$

0.70510600

$-2.98902900$

$\mathrm{H}$

0.17040700

0.37374700

$-1.87567100$

C

$-1.50708400$

$-1.68537300$

$-2.07049500$

$\mathrm{H}$

$-0.44791500$

$-1.87987100$

$-2.30176800$

$\mathrm{H}$

$-2.06709700$

$-1.63842000$

$-3.01759200$

C

$-2.02620700$

$-2.81851300$

$-1.21130900$

$\mathrm{H}$

$-1.95048000$

$-3.76236300$

$-1.77271700$

$\mathrm{H}$

$-1.42113200$

$-2.90638800$

$-0.29795400$

C

$-3.95761900$

$-3.58144900$

$-0.09013000$

$\mathrm{H}$

$-3.22758900$

$-3.99422100$

0.62102000

H

$-4.30475600$

$-4.38760700$

$-0.75157700$

C

$-5.11731500$

$-3.00358600$

0.69459600

H

$-5.60929600$

$-3.81865200$

1.25048100

$\mathrm{H}$

$-4.73598800$

$-2.27375400$

1.42363600

C

$-7.20183900$

$-1.94588600$

0.37840300

$\mathrm{H}$

$-7.56459700$

$-2.66491900$

1.13002000

$\mathrm{H}$

$-7.94388900$

$-1.88154300$

$-0.42309200$

$\mathrm{O}$

$-1.64795700$

$-0.47881000$

$-1.36124200$ 


\begin{tabular}{|c|c|c|c|}
\hline $\mathrm{O}$ & -3.36754500 & -2.55753500 & -0.86430200 \\
\hline $\mathrm{O}$ & -6.01396500 & -2.41130800 & -0.21442100 \\
\hline $\mathrm{C}$ & -7.07939100 & -0.57610400 & 1.02457800 \\
\hline $\mathrm{H}$ & -6.22450700 & -0.53682800 & 1.71742500 \\
\hline $\mathrm{H}$ & -7.99723800 & -0.37366500 & 1.60117500 \\
\hline $\mathrm{C}$ & -6.91588400 & 1.70319400 & 0.50385800 \\
\hline $\mathrm{H}$ & -7.91563300 & 1.99369300 & 0.86363100 \\
\hline $\mathrm{H}$ & -6.20525100 & 1.80009700 & 1.34079400 \\
\hline $\mathrm{C}$ & -6.47927500 & 2.61509500 & -0.62246700 \\
\hline $\mathrm{H}$ & -6.58349600 & 3.67000400 & -0.32927300 \\
\hline $\mathrm{H}$ & -7.09892700 & 2.43158500 & -1.51169900 \\
\hline $\mathrm{C}$ & -4.56234900 & 2.92874800 & -2.01995800 \\
\hline $\mathrm{H}$ & -5.22345600 & 2.83078000 & -2.89289700 \\
\hline $\mathrm{H}$ & -4.40138700 & 3.99714200 & -1.81615200 \\
\hline $\mathrm{C}$ & -3.24160600 & 2.23286500 & -2.28612800 \\
\hline $\mathrm{H}$ & -2.71507300 & 2.74129900 & -3.10910700 \\
\hline $\mathrm{H}$ & -3.42922200 & 1.18885500 & -2.57789800 \\
\hline $\mathrm{C}$ & -1.16548900 & 1.84953800 & -1.14014200 \\
\hline $\mathrm{H}$ & -0.52082300 & 2.64387000 & -1.53926700 \\
\hline $\mathrm{H}$ & -0.87867400 & 1.67792900 & -0.09970000 \\
\hline $\mathrm{O}$ & -6.92175600 & 0.38807600 & 0.00802200 \\
\hline $\mathrm{O}$ & -5.13193500 & 2.30229500 & -0.89216400 \\
\hline $\mathrm{O}$ & -2.51470300 & 2.28383300 & -1.08809600 \\
\hline $\mathrm{C}$ & 2.69944700 & -1.10409600 & 0.17956500 \\
\hline $\mathrm{C}$ & 3.54248800 & -0.84557500 & 1.24768000 \\
\hline $\mathrm{C}$ & 4.89714100 & -0.62914900 & 1.04350200 \\
\hline $\mathrm{C}$ & 5.42013800 & -0.69604200 & -0.23938400 \\
\hline $\mathrm{C}$ & 4.57639400 & -0.93925500 & -1.31412100 \\
\hline $\mathrm{C}$ & 3.21926100 & -1.13754300 & -1.10477700 \\
\hline
\end{tabular}




\begin{tabular}{llll} 
F & 3.05728700 & -0.78739100 & 2.49262300 \\
$\mathrm{~F}$ & 5.66845900 & -0.36953800 & 2.08778200 \\
$\mathrm{~F}$ & 5.04507900 & -0.98727800 & -2.55393000 \\
$\mathrm{~F}$ & 2.42761700 & -1.36107400 & -2.15180200 \\
$\mathrm{Br}$ & 7.28478500 & -0.37864000 & -0.53437900 \\
$\mathrm{Br}$ & 0.82298400 & -1.37037800 & 0.47658400 \\
$\mathrm{~S}$ & 2.91210200 & 2.33881300 & 0.44410800 \\
$\mathrm{O}$ & 3.92008700 & 1.91735300 & -0.51936600 \\
$\mathrm{O}$ & 1.50829300 & 1.99794800 & 0.07772500 \\
$\mathrm{O}$ & 3.17757300 & 1.98140000 & 1.84612500 \\
$\mathrm{O}$ & 2.90192400 & 3.96893500 & 0.37310200 \\
$\mathrm{~N}$ & 0.63565100 & 1.15838200 & 2.47090100 \\
$\mathrm{H}$ & -0.27952500 & 0.65394100 & 2.51288700 \\
$\mathrm{H}$ & 0.84317200 & 1.50686600 & 1.48257300 \\
$\mathrm{H}$ & 0.67184300 & 1.95318300 & 3.11063100 \\
$\mathrm{H}$ & 1.42045100 & 0.53755900 & 2.69918300 \\
$\mathrm{H}$ & 3.80363100 & 4.23569600 & 0.12658300 \\
\hline--------------------------------------------------------------------------------
\end{tabular}

I-II-III-IV-V of system C at M06-2X/6-31G(d)

$\mathrm{E}=-2446.1482035 \mathrm{au} \quad \mathrm{E}_{\mathrm{bsse}}=-2446.108677157127 \mathrm{au}$

No. of imaginary freq. $=0$

Coordinates (Angstroms)

$\begin{array}{llll}\text { Atoms } & \mathrm{X} & \mathrm{Y} & \mathrm{Z}\end{array}$

$\begin{array}{llll}\mathrm{C} & 3.05397200 & -0.37757800 & -1.45194700 \\ \mathrm{~N} & 3.27712600 & 0.58436300 & -2.38867300 \\ \mathrm{H} & 3.70465500 & 1.46159800 & -2.10815900\end{array}$




\begin{tabular}{|c|c|c|c|}
\hline $\mathrm{H}$ & 3.29905700 & 0.29737700 & -3.35493800 \\
\hline $\mathrm{N}$ & 3.31155300 & -0.02824500 & -0.19802500 \\
\hline $\mathrm{H}$ & 3.69088000 & 0.88966100 & 0.01025200 \\
\hline $\mathrm{H}$ & 2.97403200 & -0.59397200 & 0.58084500 \\
\hline S & 2.46181000 & -1.90785700 & -1.89878400 \\
\hline $\mathrm{C}$ & 0.56535700 & 2.21355200 & 1.63418500 \\
\hline $\mathrm{H}$ & 1.21706200 & 2.49540700 & 2.47673300 \\
\hline $\mathrm{H}$ & -0.47858300 & 2.32741200 & 1.95132900 \\
\hline $\mathrm{C}$ & 0.27526000 & -0.05240100 & 2.16594200 \\
\hline $\mathrm{H}$ & -0.82276800 & 0.00283000 & 2.13685400 \\
\hline $\mathrm{H}$ & 0.61858300 & 0.17019400 & 3.18739700 \\
\hline $\mathrm{C}$ & 0.73150900 & -1.44185500 & 1.78447800 \\
\hline $\mathrm{H}$ & 0.24318800 & -2.16559900 & 2.45182100 \\
\hline $\mathrm{H}$ & 0.44738100 & -1.67989100 & 0.74820600 \\
\hline $\mathrm{C}$ & 2.64723200 & -2.82843700 & 1.96264500 \\
\hline $\mathrm{H}$ & 2.03109400 & -3.50048100 & 1.34958800 \\
\hline $\mathrm{H}$ & 2.64272100 & -3.18647500 & 3.00149700 \\
\hline $\mathrm{C}$ & 4.05094500 & -2.83034500 & 1.39291400 \\
\hline $\mathrm{H}$ & 4.49728500 & -3.82746400 & 1.54465700 \\
\hline $\mathrm{H}$ & 3.99136000 & -2.64189600 & 0.31271600 \\
\hline $\mathrm{C}$ & 6.15412400 & -1.81387700 & 1.63728000 \\
\hline $\mathrm{H}$ & 6.56871600 & -2.83500900 & 1.62225400 \\
\hline $\mathrm{H}$ & 6.69971100 & -1.23252300 & 2.38654100 \\
\hline $\mathrm{O}$ & 0.82715300 & 0.87959200 & 1.25980400 \\
\hline $\mathrm{O}$ & 2.13596100 & -1.51021000 & 1.92572000 \\
\hline $\mathrm{O}$ & 4.81084000 & -1.84197200 & 2.05141300 \\
\hline $\mathrm{C}$ & 6.38456000 & -1.17872300 & 0.27501300 \\
\hline $\mathrm{H}$ & 5.66972900 & -1.56521100 & -0.46842300 \\
\hline $\mathrm{H}$ & 7.40057600 & -1.43696100 & -0.06811800 \\
\hline & & S23 & \\
\hline
\end{tabular}




\begin{tabular}{|c|c|c|c|}
\hline $\mathrm{C}$ & 6.48877600 & 0.85528200 & -0.84898500 \\
\hline $\mathrm{H}$ & 7.53933800 & 0.73712700 & -1.16239600 \\
\hline $\mathrm{H}$ & 5.85214600 & 0.41946100 & -1.63564100 \\
\hline $\mathrm{C}$ & 6.17626800 & 2.32744100 & -0.70047500 \\
\hline $\mathrm{H}$ & 6.51345400 & 2.88055100 & -1.58895400 \\
\hline $\mathrm{H}$ & 6.70101000 & 2.72269100 & 0.18010400 \\
\hline $\mathrm{C}$ & 4.34253000 & 3.74581000 & -0.11372600 \\
\hline $\mathrm{H}$ & 5.10499800 & 4.21581200 & 0.52242200 \\
\hline $\mathrm{H}$ & 4.16464700 & 4.38976600 & -0.98527300 \\
\hline $\mathrm{C}$ & 3.07256600 & 3.57746500 & 0.69023200 \\
\hline $\mathrm{H}$ & 2.76513100 & 4.55969700 & 1.08477500 \\
\hline $\mathrm{H}$ & 3.28049900 & 2.91419900 & 1.54290500 \\
\hline $\mathrm{C}$ & 0.77839100 & 3.12048300 & 0.43499100 \\
\hline $\mathrm{H}$ & 0.56498700 & 4.15836200 & 0.73736200 \\
\hline $\mathrm{H}$ & 0.07427900 & 2.84451100 & -0.35284200 \\
\hline $\mathrm{O}$ & 6.25916700 & 0.22265000 & 0.38123700 \\
\hline $\mathrm{O}$ & 4.77877800 & 2.46697500 & -0.52985500 \\
\hline $\mathrm{O}$ & 2.07077300 & 3.04266300 & -0.13897000 \\
\hline $\mathrm{C}$ & -2.75367200 & -1.54562200 & -0.76385100 \\
\hline $\mathrm{C}$ & -3.64446900 & -0.83012800 & -1.55314900 \\
\hline $\mathrm{C}$ & -4.68989900 & -0.12478300 & -0.96702100 \\
\hline $\mathrm{C}$ & -4.83388000 & -0.11481300 & 0.41413600 \\
\hline $\mathrm{C}$ & -3.93782100 & -0.81826000 & 1.20423600 \\
\hline $\mathrm{C}$ & -2.90913500 & -1.53693200 & 0.61387600 \\
\hline $\mathrm{F}$ & -3.51904000 & -0.80771100 & -2.87083900 \\
\hline $\mathrm{F}$ & -5.52280200 & 0.54786800 & -1.74354700 \\
\hline $\mathrm{F}$ & -4.03069500 & -0.80603700 & 2.52784100 \\
\hline $\mathrm{F}$ & -2.06799400 & -2.20289500 & 1.40234400 \\
\hline $\mathrm{Br}$ & -6.22726000 & 0.91976300 & 1.22960300 \\
\hline
\end{tabular}




$\begin{array}{llll}\mathrm{Br} & -1.30891600 & -2.51526100 & -1.56312400 \\ \mathrm{~N} & 0.13838400 & 0.68842700 & -1.64518100 \\ \mathrm{H} & 0.24879200 & -0.22128500 & -2.09731600 \\ \mathrm{H} & 0.28987400 & 0.53799900 & -0.64094300 \\ \mathrm{H} & 0.93525300 & 1.24854200 & -1.95434100 \\ \mathrm{H} & -1.16986400 & 1.36292300 & -1.77583000 \\ \mathrm{C} & -2.56324400 & 1.98010700 & -0.57944400 \\ \mathrm{O} & -2.10236600 & 1.90619400 & -1.78859600 \\ \mathrm{O} & -2.06784300 & 1.49623100 & 0.43121500 \\ \mathrm{O} & -3.71748900 & 2.67514700 & -0.53915800 \\ \mathrm{H} & -4.00265700 & 2.66944100 & 0.38917100\end{array}$

I-II-III-IV-V-VI of system A at M06-2X/6-31G(d)

$\mathrm{E}=-3410.3477017 \mathrm{au} \quad \mathrm{E}_{\mathrm{bsse}}=-3410.307173238854 \mathrm{au}$

No. of imaginary freq. $=0$

Coordinates (Angstroms)

$\begin{array}{llll}\text { Atoms } & \mathrm{X} & \mathrm{Y} & \mathrm{Z}\end{array}$

$\begin{array}{llll}\mathrm{C} & 2.84846400 & 0.37323400 & -0.82136800 \\ \mathrm{~N} & 3.17640100 & 1.65322900 & -0.52453500 \\ \mathrm{H} & 3.51715000 & 1.81372000 & 0.42103000 \\ \mathrm{H} & 2.62029800 & 2.38916500 & -0.93960300 \\ \mathrm{~N} & 3.52928700 & -0.56708900 & -0.18498400 \\ \mathrm{H} & 4.20060200 & -0.31346600 & 0.53694700 \\ \mathrm{H} & 3.16405000 & -1.51938300 & -0.17257400 \\ \mathrm{~S} & 1.68457900 & 0.00138700 & -2.02233500 \\ \mathrm{C} & 0.62136500 & -0.56638000 & 2.34413500\end{array}$




\begin{tabular}{|c|c|c|c|}
\hline $\mathrm{H}$ & 0.92008300 & -0.96746600 & 3.32700600 \\
\hline $\mathrm{H}$ & -0.47926900 & -0.58315400 & 2.29658300 \\
\hline $\mathrm{C}$ & 0.82378300 & -2.70050700 & 1.39656300 \\
\hline $\mathrm{H}$ & -0.25994600 & -2.80894500 & 1.56081900 \\
\hline $\mathrm{H}$ & 1.35289500 & -3.17912100 & 2.23532200 \\
\hline $\mathrm{C}$ & 1.18115700 & -3.38576500 & 0.09520200 \\
\hline $\mathrm{H}$ & 0.91698400 & -4.45182600 & 0.16697000 \\
\hline $\mathrm{H}$ & 0.61615400 & -2.93830800 & -0.73427300 \\
\hline $\mathrm{C}$ & 3.01469000 & -3.89698800 & -1.30653900 \\
\hline $\mathrm{H}$ & 2.24235100 & -3.86752500 & -2.08821400 \\
\hline $\mathrm{H}$ & 3.23963900 & -4.94568900 & -1.06791400 \\
\hline $\mathrm{C}$ & 4.25124400 & -3.19478300 & -1.83084300 \\
\hline $\mathrm{H}$ & 4.63770100 & -3.75818700 & -2.69597400 \\
\hline $\mathrm{H}$ & 3.97909300 & -2.18561500 & -2.17281700 \\
\hline $\mathrm{C}$ & 6.45724700 & -2.64390900 & -1.20076700 \\
\hline $\mathrm{H}$ & 6.72946500 & -3.03481800 & -2.19397400 \\
\hline $\mathrm{H}$ & 7.18941600 & -3.00948100 & -0.47451300 \\
\hline $\mathrm{O}$ & 1.17827600 & -1.34086700 & 1.30994100 \\
\hline $\mathrm{O}$ & 2.56411700 & -3.23640500 & -0.14097500 \\
\hline $\mathrm{O}$ & 5.20833400 & -3.15250500 & -0.79904100 \\
\hline $\mathrm{C}$ & 6.53399400 & -1.12622600 & -1.23157200 \\
\hline $\mathrm{H}$ & 5.69237600 & -0.69755400 & -1.79726100 \\
\hline $\mathrm{H}$ & 7.47055900 & -0.82880900 & -1.73165100 \\
\hline $\mathrm{C}$ & 6.67852300 & 0.74892700 & 0.16815500 \\
\hline $\mathrm{H}$ & 7.69854500 & 1.04617400 & -0.12239800 \\
\hline $\mathrm{H}$ & 5.96595700 & 1.25780600 & -0.50221200 \\
\hline $\mathrm{C}$ & 6.39876600 & 1.16701400 & 1.59588500 \\
\hline $\mathrm{H}$ & 6.63275700 & 2.23199000 & 1.74082100 \\
\hline $\mathrm{H}$ & 7.01400500 & 0.57153200 & 2.28509400 \\
\hline
\end{tabular}




\begin{tabular}{|c|c|c|c|}
\hline $\mathrm{C}$ & 4.58768100 & 1.06638400 & 3.15710800 \\
\hline $\mathrm{H}$ & 5.25561100 & 0.53243600 & 3.84790600 \\
\hline $\mathrm{H}$ & 4.56960800 & 2.13085900 & 3.43382700 \\
\hline $\mathrm{C}$ & 3.19120900 & 0.47735400 & 3.22013400 \\
\hline $\mathrm{H}$ & 2.76009100 & 0.64738600 & 4.21899500 \\
\hline $\mathrm{H}$ & 3.23729000 & -0.60544400 & 3.03290300 \\
\hline $\mathrm{C}$ & 1.05483800 & 0.87875100 & 2.18850900 \\
\hline $\mathrm{H}$ & 0.54807800 & 1.47987400 & 2.95612100 \\
\hline $\mathrm{H}$ & 0.73163800 & 1.23869900 & 1.20759500 \\
\hline $\mathrm{O}$ & 6.51958600 & -0.64552900 & 0.09435500 \\
\hline $\mathrm{O}$ & 5.02982400 & 0.92108800 & 1.82579100 \\
\hline $\mathrm{O}$ & 2.45071800 & 1.11580700 & 2.21397100 \\
\hline $\mathrm{C}$ & -3.19595900 & -0.57698800 & -0.19269800 \\
\hline $\mathrm{C}$ & -3.94369000 & 0.30472300 & -0.95520100 \\
\hline $\mathrm{C}$ & -5.27312800 & 0.55256500 & -0.64694500 \\
\hline $\mathrm{C}$ & -5.86861300 & -0.10320500 & 0.42105700 \\
\hline $\mathrm{C}$ & -5.11801500 & -0.97767700 & 1.19516500 \\
\hline $\mathrm{C}$ & -3.78361500 & -1.20830200 & 0.89160900 \\
\hline $\mathrm{F}$ & -3.39138100 & 0.93864300 & -1.99396200 \\
\hline $\mathrm{F}$ & -5.95361600 & 1.41083300 & -1.39209100 \\
\hline $\mathrm{F}$ & -5.65672900 & -1.60835900 & 2.23051400 \\
\hline $\mathrm{F}$ & -3.08102300 & -2.04382900 & 1.65368400 \\
\hline $\mathrm{Br}$ & -7.70214700 & 0.23524200 & 0.85606600 \\
\hline $\mathrm{Br}$ & -1.35228800 & -0.88597100 & -0.62019500 \\
\hline $\mathrm{O}$ & -3.94876400 & 1.73127500 & 1.92931500 \\
\hline $\mathrm{O}$ & -1.58144100 & 1.83776300 & 1.46563800 \\
\hline $\mathrm{O}$ & -3.12679600 & 2.89464900 & -0.02666500 \\
\hline $\mathrm{O}$ & -2.76660400 & 3.82083300 & 2.17982000 \\
\hline $\mathrm{N}$ & -0.59751300 & 2.23512600 & -1.13145600 \\
\hline
\end{tabular}




\begin{tabular}{|c|c|c|c|}
\hline $\mathrm{H}$ & 0.13190000 & 1.55620800 & -1.46193700 \\
\hline $\mathrm{H}$ & -0.80937300 & 2.08999200 & -0.11695000 \\
\hline $\mathrm{H}$ & -0.28799900 & 3.20730700 & -1.27509600 \\
\hline $\mathrm{H}$ & -1.49113600 & 2.07418500 & -1.61028800 \\
\hline $\mathrm{C}$ & 0.49106100 & 5.41243700 & -1.98480300 \\
\hline $\mathrm{C}$ & 1.82906700 & 5.10937800 & -2.28801800 \\
\hline $\mathrm{C}$ & 2.73678700 & 4.84097900 & -1.24954000 \\
\hline $\mathrm{C}$ & 2.27435600 & 4.73225600 & 0.07600200 \\
\hline $\mathrm{C}$ & 0.92297600 & 4.98123300 & 0.37532500 \\
\hline $\mathrm{C}$ & 0.03368600 & 5.34365700 & -0.65492500 \\
\hline $\mathrm{Cl}$ & -2.88702000 & 2.58605400 & 1.40446900 \\
\hline $\mathrm{C}$ & 0.41671800 & 4.88436900 & 1.79771500 \\
\hline $\mathrm{H}$ & -0.21479000 & 5.73993900 & 2.05039500 \\
\hline $\mathrm{H}$ & -0.20039400 & 3.98932200 & 1.94808600 \\
\hline $\mathrm{H}$ & 1.23181700 & 4.85593600 & 2.52052300 \\
\hline $\mathrm{C}$ & 2.29046500 & 5.07495400 & -3.72919100 \\
\hline $\mathrm{H}$ & 2.69381100 & 6.04159200 & -4.05726300 \\
\hline $\mathrm{H}$ & 3.06991300 & 4.32440900 & -3.87636900 \\
\hline $\mathrm{H}$ & 1.47038700 & 4.81705400 & -4.40153600 \\
\hline $\mathrm{C}$ & -1.41860100 & 5.64147300 & -0.34859200 \\
\hline $\mathrm{H}$ & -1.73798700 & 5.23762100 & 0.61150400 \\
\hline $\mathrm{H}$ & -1.59618400 & 6.72476700 & -0.33414900 \\
\hline $\mathrm{H}$ & -2.08875100 & 5.21378500 & -1.09947500 \\
\hline $\mathrm{C}$ & -0.45816300 & 5.84225000 & -3.08375400 \\
\hline $\mathrm{H}$ & -0.97341500 & 4.99004200 & -3.54741600 \\
\hline $\mathrm{H}$ & -1.22752000 & 6.51336100 & -2.69953000 \\
\hline $\mathrm{H}$ & 0.06907200 & 6.37346100 & -3.87839400 \\
\hline $\mathrm{C}$ & 4.21415400 & 4.70035400 & -1.55885800 \\
\hline $\mathrm{H}$ & 4.52828200 & 3.65070900 & -1.62920100 \\
\hline
\end{tabular}




$\begin{array}{llll}\mathrm{H} & 4.46954800 & 5.18752500 & -2.50058300 \\ \mathrm{H} & 4.81885200 & 5.17512900 & -0.78259200 \\ \mathrm{C} & 3.22305900 & 4.33231200 & 1.18874500 \\ \mathrm{H} & 4.17653200 & 3.96557000 & 0.80233900 \\ \mathrm{H} & 3.44127300 & 5.17589900 & 1.85468900 \\ \mathrm{H} & 2.79395600 & 3.53121300 & 1.80002600\end{array}$

\section{I-II-III-IV-V-VI of system B at M06-2X/6-31G(d)}
$E=-3349.2541407 \mathrm{au}$
$E_{b s s e}=-3349.211764674887 \mathrm{au}$

No. of imaginary freq. $=0$

Coordinates (Angstroms)

$\begin{array}{llll}\text { Atoms } & \mathrm{X} & \mathrm{Y} & \mathrm{Z}\end{array}$

$\begin{array}{cccc}\mathrm{C} & 3.10988200 & 0.35313100 & -0.72271600 \\ \mathrm{~N} & 3.48990700 & 1.60945300 & -0.37985100 \\ \mathrm{H} & 3.80463100 & 1.71741800 & 0.58206000 \\ \mathrm{H} & 2.91751700 & 2.36071800 & -0.74207900 \\ \mathrm{~N} & 3.72081200 & -0.63684100 & -0.08709300 \\ \mathrm{H} & 4.34325600 & -0.43585500 & 0.69306400 \\ \mathrm{H} & 3.29949000 & -1.56506600 & -0.11227800 \\ \mathrm{~S} & 1.98081200 & 0.07152600 & -1.97733900 \\ \mathrm{C} & 0.64735700 & -0.41172500 & 2.27537100 \\ \mathrm{H} & 0.83778700 & -0.83474500 & 3.27599200 \\ \mathrm{H} & -0.44319000 & -0.34122700 & 2.14092800 \\ \mathrm{C} & 0.79569000 & -2.56705600 & 1.37031000 \\ \mathrm{H} & -0.29929600 & -2.61717800 & 1.48007100 \\ \mathrm{H} & 1.25489700 & -3.05883400 & 2.24232100 \\ & & \mathrm{~S} 29 & \end{array}$




\begin{tabular}{|c|c|c|c|}
\hline $\mathrm{C}$ & 1.17839200 & -3.29649600 & 0.10043400 \\
\hline $\mathrm{H}$ & 0.84393300 & -4.34263800 & 0.17545400 \\
\hline $\mathrm{H}$ & 0.68606400 & -2.83217400 & -0.76507600 \\
\hline $\mathrm{C}$ & 3.03794100 & -3.95078400 & -1.19927400 \\
\hline $\mathrm{H}$ & 2.31563000 & -3.87027900 & -2.02413300 \\
\hline $\mathrm{H}$ & 3.16365100 & -5.01098000 & -0.93886100 \\
\hline $\mathrm{C}$ & 4.35497000 & -3.35719100 & -1.65674300 \\
\hline $\mathrm{H}$ & 4.74233400 & -3.95819400 & -2.49582000 \\
\hline $\mathrm{H}$ & 4.18647800 & -2.33244800 & -2.01891700 \\
\hline $\mathrm{C}$ & 6.55538100 & -2.96796000 & -0.89882300 \\
\hline $\mathrm{H}$ & 6.85432500 & -3.37335900 & -1.87846600 \\
\hline $\mathrm{H}$ & 7.21584300 & -3.39001100 & -0.13539300 \\
\hline $\mathrm{O}$ & 1.22466600 & -1.22954100 & 1.28428000 \\
\hline $\mathrm{O}$ & 2.57775800 & -3.23980700 & -0.06798000 \\
\hline $\mathrm{O}$ & 5.25139600 & -3.38341200 & -0.57186400 \\
\hline $\mathrm{C}$ & 6.74227600 & -1.46005000 & -0.91053700 \\
\hline $\mathrm{H}$ & 5.97323500 & -0.96970400 & -1.52679800 \\
\hline $\mathrm{H}$ & 7.72939100 & -1.22779000 & -1.34370100 \\
\hline $\mathrm{C}$ & 6.91346600 & 0.39282400 & 0.51419600 \\
\hline $\mathrm{H}$ & 7.96636600 & 0.62592300 & 0.28948700 \\
\hline $\mathrm{H}$ & 6.27791200 & 0.95084600 & -0.19318300 \\
\hline $\mathrm{C}$ & 6.57424200 & 0.81895500 & 1.92656900 \\
\hline $\mathrm{H}$ & 6.87272500 & 1.86356100 & 2.09811200 \\
\hline $\mathrm{H}$ & 7.10194100 & 0.17650500 & 2.64564500 \\
\hline $\mathrm{C}$ & 4.66617600 & 0.85940900 & 3.36849500 \\
\hline $\mathrm{H}$ & 5.23409500 & 0.26705000 & 4.10008700 \\
\hline $\mathrm{H}$ & 4.72945000 & 1.92158200 & 3.64722600 \\
\hline $\mathrm{C}$ & 3.21955900 & 0.40567300 & 3.33724900 \\
\hline $\mathrm{H}$ & 2.74634000 & 0.60234500 & 4.31195200 \\
\hline
\end{tabular}




\begin{tabular}{|c|c|c|c|}
\hline $\mathrm{H}$ & 3.17652900 & -0.67400700 & 3.13260500 \\
\hline $\mathrm{C}$ & 1.19908700 & 0.99662900 & 2.16759400 \\
\hline $\mathrm{H}$ & 0.68153300 & 1.62840300 & 2.90180600 \\
\hline $\mathrm{H}$ & 0.97826800 & 1.39140700 & 1.17201000 \\
\hline $\mathrm{O}$ & 6.67125800 & -0.98789300 & 0.41620900 \\
\hline $\mathrm{O}$ & 5.17985300 & 0.66962600 & 2.06912700 \\
\hline $\mathrm{O}$ & 2.60499000 & 1.12441900 & 2.30127100 \\
\hline $\mathrm{C}$ & -3.06234600 & -0.37356100 & -0.51441900 \\
\hline $\mathrm{C}$ & -3.69883000 & 0.59430400 & -1.27427100 \\
\hline $\mathrm{C}$ & -5.03866600 & 0.88775700 & -1.06691900 \\
\hline $\mathrm{C}$ & -5.75647000 & 0.18990500 & -0.10628900 \\
\hline $\mathrm{C}$ & -5.12148600 & -0.77869800 & 0.65912500 \\
\hline $\mathrm{C}$ & -3.77622100 & -1.05315600 & 0.45992600 \\
\hline $\mathrm{F}$ & -3.03573000 & 1.25317400 & -2.22744700 \\
\hline $\mathrm{F}$ & -5.61197900 & 1.82726200 & -1.80342500 \\
\hline $\mathrm{F}$ & -5.78156200 & -1.45051100 & 1.59542900 \\
\hline $\mathrm{F}$ & -3.18734900 & -1.97736500 & 1.21427300 \\
\hline $\mathrm{Br}$ & -7.60481100 & 0.59746200 & 0.19831700 \\
\hline $\mathrm{Br}$ & -1.20451500 & -0.75023500 & -0.80036500 \\
\hline S & -2.61542100 & 2.80599900 & 1.23794600 \\
\hline $\mathrm{O}$ & -3.82113900 & 1.77595900 & 1.65425900 \\
\hline $\mathrm{O}$ & -1.39672400 & 1.96984600 & 1.38137000 \\
\hline $\mathrm{O}$ & -2.84971300 & 3.15253500 & -0.17322800 \\
\hline $\mathrm{O}$ & -2.73904200 & 3.89725000 & 2.19860500 \\
\hline $\mathrm{N}$ & -0.33466600 & 2.33849300 & -1.13013800 \\
\hline $\mathrm{H}$ & 0.40111100 & 1.67566500 & -1.45738600 \\
\hline $\mathrm{H}$ & -0.57156700 & 2.17823600 & -0.11874300 \\
\hline $\mathrm{H}$ & -0.04150300 & 3.31512500 & -1.26194300 \\
\hline $\mathrm{H}$ & -1.21984600 & 2.19119900 & -1.62798300 \\
\hline
\end{tabular}




\begin{tabular}{|c|c|c|c|}
\hline $\mathrm{C}$ & -0.16413200 & 5.57337200 & -1.56541600 \\
\hline $\mathrm{C}$ & 1.02999500 & 5.13154400 & -2.15317000 \\
\hline $\mathrm{C}$ & 2.12747900 & 4.76792900 & -1.33713700 \\
\hline $\mathrm{C}$ & 1.97292300 & 4.72901700 & 0.06074700 \\
\hline $\mathrm{C}$ & 0.73143700 & 5.07786200 & 0.64308600 \\
\hline $\mathrm{C}$ & -0.30684400 & 5.56712900 & -0.16083700 \\
\hline $\mathrm{H}$ & -4.41208300 & 2.28450200 & 2.23504600 \\
\hline $\mathrm{C}$ & 3.11021100 & 4.37979300 & 1.00185700 \\
\hline $\mathrm{H}$ & 3.25849500 & 5.18622500 & 1.72773800 \\
\hline $\mathrm{H}$ & 2.89700900 & 3.47102700 & 1.57744300 \\
\hline $\mathrm{H}$ & 4.05936200 & 4.22649400 & 0.48936200 \\
\hline $\mathrm{C}$ & 3.44194800 & 4.46559600 & -2.03288900 \\
\hline $\mathrm{H}$ & 4.28709800 & 4.42207700 & -1.34828700 \\
\hline $\mathrm{H}$ & 3.41613300 & 3.51036400 & -2.57275200 \\
\hline $\mathrm{H}$ & 3.66498000 & 5.24160100 & -2.77138800 \\
\hline $\mathrm{C}$ & 1.16851200 & 5.05398400 & -3.65952300 \\
\hline $\mathrm{H}$ & 1.49903100 & 6.00952700 & -4.08621700 \\
\hline $\mathrm{H}$ & 1.89414600 & 4.29574800 & -3.95780800 \\
\hline $\mathrm{H}$ & 0.22311300 & 4.79346100 & -4.13821700 \\
\hline $\mathrm{C}$ & -1.33758100 & 6.04745600 & -2.39080700 \\
\hline $\mathrm{H}$ & -1.10602600 & 6.13509100 & -3.45127600 \\
\hline $\mathrm{H}$ & -2.19165400 & 5.36855100 & -2.27391000 \\
\hline $\mathrm{H}$ & -1.67329100 & 7.03135300 & -2.04951500 \\
\hline $\mathrm{C}$ & -1.57430800 & 6.13293500 & 0.43274700 \\
\hline $\mathrm{H}$ & -1.67912500 & 7.18458900 & 0.13943500 \\
\hline $\mathrm{H}$ & -2.45626700 & 5.59297600 & 0.07562200 \\
\hline $\mathrm{H}$ & -1.58996000 & 6.08134000 & 1.51847400 \\
\hline $\mathrm{C}$ & 0.53399200 & 4.96763000 & 2.13978300 \\
\hline $\mathrm{H}$ & 0.64689700 & 5.94372900 & 2.62879300 \\
\hline
\end{tabular}




$\begin{array}{llll}\mathrm{H} & -0.46339800 & 4.58812000 & 2.37576700 \\ \mathrm{H} & 1.25600900 & 4.28899700 & 2.59527700\end{array}$

I-II-III-IV-V-VI of system C at M06-2X/6-31G(d)

$\mathrm{E}=-2914.0729785 \mathrm{au} \quad \mathrm{E}_{\mathrm{bsse}}=-2914.020154725828 \mathrm{au}$

No. of imaginary freq. $=0$

Coordinates (Angstroms)

\begin{tabular}{|c|c|c|c|}
\hline Atoms & $X$ & $\mathrm{Y}$ & $\mathrm{Z}$ \\
\hline $\mathrm{C}$ & 2.85021800 & 0.29497600 & -1.16338600 \\
\hline $\mathrm{N}$ & 3.14016100 & 1.61658900 & -1.10185600 \\
\hline $\mathrm{H}$ & 3.39875300 & 2.02616200 & -0.20786800 \\
\hline $\mathrm{H}$ & 2.72513300 & 2.22076700 & -1.79676200 \\
\hline $\mathrm{N}$ & 3.25839100 & -0.44025700 & -0.13827300 \\
\hline $\mathrm{H}$ & 3.80177800 & -0.01918000 & 0.60886900 \\
\hline $\mathrm{H}$ & 2.92032400 & -1.39481700 & -0.01556400 \\
\hline $\mathrm{S}$ & 2.02630000 & -0.34760100 & -2.51399700 \\
\hline $\mathrm{C}$ & 0.52195700 & -0.22133200 & 2.85231400 \\
\hline $\mathrm{H}$ & 1.18241300 & -0.70015100 & 3.59158600 \\
\hline $\mathrm{H}$ & -0.51999200 & -0.37827100 & 3.15154800 \\
\hline $\mathrm{C}$ & 0.15296800 & -2.06328800 & 1.44072100 \\
\hline $\mathrm{H}$ & -0.93909800 & -1.95154900 & 1.46364900 \\
\hline $\mathrm{H}$ & 0.47250800 & -2.70964600 & 2.27088600 \\
\hline $\mathrm{C}$ & 0.58165000 & -2.69098100 & 0.13454600 \\
\hline $\mathrm{H}$ & 0.03807100 & -3.63860300 & 0.01583200 \\
\hline $\mathrm{H}$ & 0.33681700 & -2.04183400 & -0.71948400 \\
\hline $\mathrm{C}$ & 2.45597100 & -3.82572700 & -0.80366000 \\
\hline
\end{tabular}




\begin{tabular}{|c|c|c|c|}
\hline $\mathrm{H}$ & 1.80282200 & -3.82137000 & -1.68627100 \\
\hline $\mathrm{H}$ & 2.47767400 & -4.83813400 & -0.37832400 \\
\hline $\mathrm{C}$ & 3.84315900 & -3.39754500 & -1.24658000 \\
\hline $\mathrm{H}$ & 4.26936400 & -4.18308700 & -1.89230600 \\
\hline $\mathrm{H}$ & 3.75806100 & -2.47557200 & -1.83742400 \\
\hline $\mathrm{C}$ & 5.98520500 & -2.89061000 & -0.41074100 \\
\hline $\mathrm{H}$ & 6.36357700 & -3.56923900 & -1.19195800 \\
\hline $\mathrm{H}$ & 6.56295600 & -3.06259800 & 0.50229800 \\
\hline $\mathrm{O}$ & 0.76082800 & -0.78835600 & 1.57853200 \\
\hline $\mathrm{O}$ & 1.97718400 & -2.92545600 & 0.17806600 \\
\hline $\mathrm{O}$ & 4.64821800 & -3.20586600 & -0.10381200 \\
\hline $\mathrm{C}$ & 6.21834000 & -1.45634700 & -0.86315800 \\
\hline $\mathrm{H}$ & 5.47029800 & -1.15358800 & -1.61205100 \\
\hline $\mathrm{H}$ & 7.21322800 & -1.39163700 & -1.33419500 \\
\hline $\mathrm{C}$ & 6.35908000 & 0.75065500 & -0.11413200 \\
\hline $\mathrm{H}$ & 7.39018900 & 0.91507700 & -0.46680500 \\
\hline $\mathrm{H}$ & 5.66998600 & 1.03976100 & -0.92316200 \\
\hline $\mathrm{C}$ & 6.10143500 & 1.61519500 & 1.10054500 \\
\hline $\mathrm{H}$ & 6.39271600 & 2.65588500 & 0.89954300 \\
\hline $\mathrm{H}$ & 6.69170200 & 1.23862300 & 1.94716200 \\
\hline $\mathrm{C}$ & 4.35695400 & 1.95355100 & 2.70633300 \\
\hline $\mathrm{H}$ & 5.14174900 & 1.67615800 & 3.42411300 \\
\hline $\mathrm{H}$ & 4.22565800 & 3.04418000 & 2.73662600 \\
\hline $\mathrm{C}$ & 3.07943600 & 1.24517900 & 3.09932200 \\
\hline $\mathrm{H}$ & 2.85408700 & 1.48335300 & 4.15139300 \\
\hline $\mathrm{H}$ & 3.23806300 & 0.16005900 & 3.01865400 \\
\hline $\mathrm{C}$ & 0.75162900 & 1.27978300 & 2.78716100 \\
\hline $\mathrm{H}$ & 0.62218700 & 1.69865500 & 3.79777100 \\
\hline $\mathrm{H}$ & -0.00200800 & 1.73540100 & 2.13925800 \\
\hline
\end{tabular}




\begin{tabular}{|c|c|c|c|}
\hline $\mathrm{O}$ & 6.16336100 & -0.59273700 & 0.25225800 \\
\hline $\mathrm{O}$ & 4.72128800 & 1.53611900 & 1.40782200 \\
\hline $\mathrm{O}$ & 2.01797300 & 1.65752900 & 2.26827100 \\
\hline $\mathrm{C}$ & -3.10365100 & -0.55239600 & -1.52306900 \\
\hline $\mathrm{C}$ & -4.06580500 & 0.43558600 & -1.36727900 \\
\hline $\mathrm{C}$ & -5.04355300 & 0.30751100 & -0.38920200 \\
\hline $\mathrm{C}$ & -5.05106900 & -0.80250300 & 0.44592500 \\
\hline $\mathrm{C}$ & -4.08387300 & -1.78370600 & 0.29668200 \\
\hline $\mathrm{C}$ & -3.11864400 & -1.65993400 & -0.69119600 \\
\hline $\mathrm{F}$ & -4.05788800 & 1.51724800 & -2.13061300 \\
\hline $\mathrm{F}$ & -5.94189300 & 1.26844800 & -0.25200300 \\
\hline $\mathrm{F}$ & -4.03960700 & -2.83833600 & 1.10240000 \\
\hline $\mathrm{F}$ & -2.19356700 & -2.61239400 & -0.79793900 \\
\hline $\mathrm{Br}$ & -6.34570000 & -0.93436200 & 1.85322700 \\
\hline $\mathrm{Br}$ & -1.71974400 & -0.35625200 & -2.83318800 \\
\hline $\mathrm{N}$ & 0.08572300 & 1.26285700 & -0.32801500 \\
\hline $\mathrm{H}$ & 0.25744500 & 0.97962400 & -1.30212800 \\
\hline $\mathrm{H}$ & 0.27435000 & 0.44476200 & 0.28145600 \\
\hline $\mathrm{H}$ & 0.75093600 & 2.00608100 & -0.09068400 \\
\hline $\mathrm{H}$ & -0.95367700 & 1.60147800 & -0.13715000 \\
\hline $\mathrm{C}$ & 1.05069200 & 4.26681100 & -2.32854000 \\
\hline $\mathrm{C}$ & 2.40669400 & 4.48640900 & -2.04038700 \\
\hline $\mathrm{C}$ & 2.82015700 & 4.65758300 & -0.70743200 \\
\hline $\mathrm{C}$ & 1.86381000 & 4.73094800 & 0.31643600 \\
\hline $\mathrm{C}$ & 0.49646300 & 4.56452400 & 0.02209800 \\
\hline $\mathrm{C}$ & 0.08799800 & 4.33834800 & -1.30416800 \\
\hline $\mathrm{C}$ & -2.63123700 & 1.24388500 & 1.24306000 \\
\hline $\mathrm{O}$ & -2.29133400 & 2.04575300 & 0.32355500 \\
\hline $\mathrm{O}$ & -2.06698000 & 0.18645200 & 1.57221700 \\
\hline
\end{tabular}




\begin{tabular}{|c|c|c|c|}
\hline $\mathrm{O}$ & -3.75869800 & 1.62097700 & 1.91643500 \\
\hline $\mathrm{H}$ & -3.91801400 & 0.91955500 & 2.56768100 \\
\hline $\mathrm{C}$ & -0.52139500 & 4.62593400 & 1.14100100 \\
\hline $\mathrm{H}$ & -0.94009800 & 5.63617800 & 1.23962900 \\
\hline $\mathrm{H}$ & -1.34945100 & 3.93351800 & 0.97354400 \\
\hline $\mathrm{H}$ & -0.07111200 & 4.37012800 & 2.10280500 \\
\hline $\mathrm{C}$ & 2.27680700 & 5.03393200 & 1.74048900 \\
\hline $\mathrm{H}$ & 1.63607000 & 5.80981400 & 2.16936600 \\
\hline $\mathrm{H}$ & 2.18629000 & 4.14410100 & 2.37544500 \\
\hline $\mathrm{H}$ & 3.30379700 & 5.39729800 & 1.80210800 \\
\hline $\mathrm{C}$ & 4.29882600 & 4.68590400 & -0.37519500 \\
\hline $\mathrm{H}$ & 4.69529900 & 5.70512200 & -0.28544100 \\
\hline $\mathrm{H}$ & 4.49112300 & 4.17353700 & 0.57314100 \\
\hline $\mathrm{H}$ & 4.88595300 & 4.16762200 & -1.13772000 \\
\hline $\mathrm{C}$ & 3.42474000 & 4.51506300 & -3.16222700 \\
\hline $\mathrm{H}$ & 4.24606000 & 5.19684300 & -2.93112500 \\
\hline $\mathrm{H}$ & 3.86380100 & 3.52629800 & -3.35765900 \\
\hline $\mathrm{H}$ & 2.97307300 & 4.85947200 & -4.09405100 \\
\hline $\mathrm{C}$ & 0.61934300 & 3.93837900 & -3.74140000 \\
\hline $\mathrm{H}$ & 0.41762300 & 4.84346400 & -4.32905300 \\
\hline $\mathrm{H}$ & 1.38420400 & 3.36342000 & -4.26872000 \\
\hline $\mathrm{H}$ & -0.29160800 & 3.33695000 & -3.74556100 \\
\hline $\mathrm{C}$ & -1.37683400 & 4.20824800 & -1.66300100 \\
\hline $\mathrm{H}$ & -1.60124700 & 4.79986400 & -2.55581700 \\
\hline $\mathrm{H}$ & -1.66977700 & 3.17224200 & -1.87358000 \\
\hline $\mathrm{H}$ & -2.02925700 & 4.54989900 & -0.86116600 \\
\hline
\end{tabular}


2. M06-2X/6-31G(d)/ECP optimized structures of the 1:1:1:1:1:1 molecular complexes:

(i)

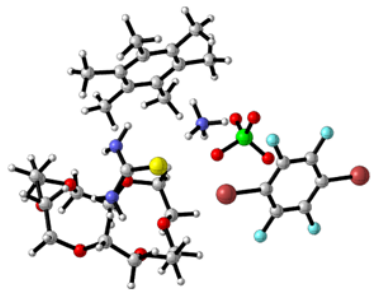

(ii)

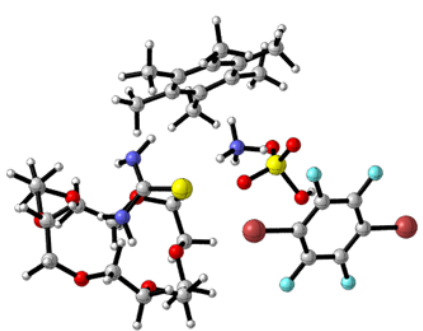

(iii)

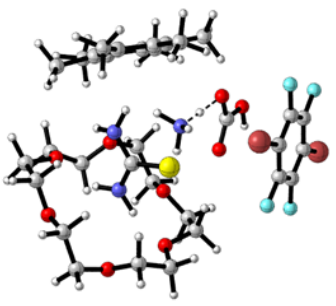

Figure S1. M06-2X/6-31G(d)/ECP optimized 1:1:1:1:1:1 molecular complex clearly indicating the stability of $H$-bridging pair in case of $C$.

3. Table ST1: Range-separation parameters for the 6-component molecular complex and its individual components:

\begin{tabular}{|c|c|c|c|c|}
\hline System & $\boldsymbol{\alpha}$ & $\boldsymbol{\beta}$ & $\boldsymbol{\gamma}$ & $\boldsymbol{J}^{\mathbf{2}}$ \\
\hline Crownether (I) & 0.1818 & 0.8182 & 0.1616 & 0.002 \\
\hline Thiourea (II) & 0.3838 & 0.6162 & 0.2424 & 0.010 \\
\hline $\begin{array}{c}\text { 2,3,5,6-tetrafluro-1,4- } \\
\text { dibromobenzene (III) }\end{array}$ & 0.4646 & 0.5354 & 0.1818 & 0.004 \\
\hline Ammonium (IV) & 0.4091 & 0.5909 & 0.3737 & 0.040 \\
\hline Perchlorate (V) & 0.3586 & 0.6414 & 0.1566 & 0.015 \\
\hline Bisulfate (V) & 0.3838 & 0.6162 & 0.1465 & 0.005 \\
\hline Bicarbonate (V) & 0.3990 & 0.6010 & 0.1667 & 0.010 \\
\hline Hexamethylbenzene (VI) & 0.3333 & 0.6667 & 0.1566 & 0.005 \\
\hline Ammonium-Perchlorate & 0.3737 & 0.6263 & 0.1515 & 0.006 \\
\hline Ammonium-Bisulfate & 0.4545 & 0.5455 & 0.3182 & 0.062 \\
\hline Ammonium-Bicarbonate & 0.3636 & 0.6364 & 0.1768 & 0.032 \\
\hline Complex A & 0.2677 & 0.7323 & 0.1616 & 0.004 \\
\hline Complex B & 0.4596 & 0.5404 & 0.1162 & 0.002 \\
\hline Complex C & 0.2273 & 0.7727 & 0.2273 & 0.007 \\
\hline
\end{tabular}

\section{Optimized geometries and cell parameters from Periodic DFT calculations using Crystal17:}

\section{Crystal A:}

Cell parameters:
$a=17.0123906 \AA$
$\mathrm{b}=11.5507628 \AA$
$c=8.0040124 \AA$
$\alpha=104.236578$
$\beta=102.685607$
$\gamma=85.065624$ 
Coordinates (Angstroms)

$\begin{array}{llll}\text { Atoms } & \mathrm{X} & \mathrm{Y} & \mathrm{Z}\end{array}$

\begin{tabular}{|c|c|c|c|}
\hline $\mathrm{H}$ & 10.89896822 & 5.46794438 & 4.98986432 \\
\hline $\mathrm{H}$ & 9.83595375 & 6.13739267 & 3.80961714 \\
\hline $\mathrm{H}$ & 12.28360582 & 3.80047726 & 4.50651197 \\
\hline $\mathrm{H}$ & 11.81414772 & 2.72037415 & 3.23596582 \\
\hline $\mathrm{H}$ & 9.42318604 & 1.68524015 & 6.65552106 \\
\hline $\mathrm{H}$ & 7.93568366 & 2.06902775 & 5.77123249 \\
\hline $\mathrm{H}$ & 8.42474072 & 0.51367924 & 4.22411588 \\
\hline $\mathrm{H}$ & 10.05977103 & 0.08520748 & 4.78457179 \\
\hline $\mathrm{H}$ & 9.75790299 & -0.33141174 & 2.38096953 \\
\hline $\mathrm{H}$ & 9.28620605 & 1.37482221 & 2.10864036 \\
\hline $\mathrm{H}$ & 11.01603182 & 1.29120775 & 0.55526808 \\
\hline $\mathrm{H}$ & 12.01851360 & -0.09596154 & 1.07973519 \\
\hline $\mathrm{H}$ & 13.46599049 & 1.66527589 & 0.17827786 \\
\hline $\mathrm{H}$ & 12.64945869 & 2.89651438 & 1.17694205 \\
\hline $\mathrm{H}$ & 15.47194864 & 2.31484960 & 1.15156205 \\
\hline $\mathrm{H}$ & 15.77421529 & 1.76702321 & 2.81533602 \\
\hline $\mathrm{H}$ & 14.12107582 & 4.25275094 & 2.16019170 \\
\hline $\mathrm{H}$ & 15.89358010 & 4.25489098 & 2.40870477 \\
\hline $\mathrm{H}$ & 15.63556894 & 5.60598460 & 4.39635710 \\
\hline $\mathrm{H}$ & 13.89251941 & 5.64496683 & 3.99155479 \\
\hline $\mathrm{H}$ & 14.40220732 & 6.05299543 & 6.44104438 \\
\hline $\mathrm{H}$ & 15.00918646 & 4.37730751 & 6.53771555 \\
\hline $\mathrm{H}$ & 15.01580108 & 5.57758289 & 0.39170878 \\
\hline $\mathrm{H}$ & 14.08986155 & 7.10423142 & 0.33231498 \\
\hline $\mathrm{H}$ & 12.55403006 & 5.15540465 & 0.66286657 \\
\hline
\end{tabular}




\begin{tabular}{|c|c|c|c|}
\hline $\mathrm{H}$ & 11.46686240 & 2.57444559 & 6.78091958 \\
\hline $\mathrm{H}$ & 8.44560995 & 3.94484334 & 7.11881382 \\
\hline $\mathrm{H}$ & 8.66282345 & 4.38930153 & 5.41286002 \\
\hline $\mathrm{H}$ & 8.42301880 & 5.68135347 & 1.64149798 \\
\hline $\mathrm{H}$ & 7.95091981 & 7.26471782 & 1.96526863 \\
\hline $\mathrm{H}$ & 7.54085224 & 6.57705552 & 0.48720690 \\
\hline $\mathrm{H}$ & 6.78905718 & 6.07556741 & 1.94290139 \\
\hline $\mathrm{H}$ & 7.32186959 & -0.74860009 & 5.88363160 \\
\hline $\mathrm{H}$ & 7.80442775 & 9.08613477 & 6.09958422 \\
\hline $\mathrm{H}$ & 9.45246807 & 9.57936128 & 6.53527285 \\
\hline $\mathrm{H}$ & 8.84386769 & 7.58519954 & 7.42189225 \\
\hline $\mathrm{H}$ & 10.90914457 & 7.72903268 & 0.25346567 \\
\hline $\mathrm{H}$ & 7.54982639 & 6.41050565 & 7.31628914 \\
\hline $\mathrm{H}$ & 6.39614133 & 9.83831983 & 4.52367815 \\
\hline $\mathrm{H}$ & 6.02816461 & -0.09096343 & 4.03012201 \\
\hline $\mathrm{H}$ & 6.18882291 & 10.37921644 & 2.86398975 \\
\hline $\mathrm{H}$ & 6.88976725 & 9.24978924 & 0.64381764 \\
\hline $\mathrm{H}$ & 7.21558563 & 10.90847441 & 1.13959918 \\
\hline $\mathrm{H}$ & 6.50045937 & 8.28204699 & 7.56977963 \\
\hline $\mathrm{H}$ & 11.97144587 & 6.92837686 & 2.08713168 \\
\hline $\mathrm{H}$ & 12.47042271 & 8.55369010 & 1.60010839 \\
\hline $\mathrm{H}$ & 12.75169122 & 8.01127080 & 3.24660276 \\
\hline $\mathrm{H}$ & 12.02591066 & 7.73790172 & 4.98724278 \\
\hline $\mathrm{H}$ & 11.62694551 & 9.25779078 & 5.79683578 \\
\hline $\mathrm{H}$ & 10.62936515 & 7.82094644 & 6.03991327 \\
\hline $\mathrm{C}$ & 10.87065259 & 4.49552104 & 3.23260588 \\
\hline $\mathrm{C}$ & 9.00251413 & 2.32479134 & 5.86156832 \\
\hline $\mathrm{C}$ & 9.48633789 & 0.79826173 & 4.16798551 \\
\hline $\mathrm{C}$ & 9.90592117 & 0.70370580 & 2.72006656 \\
\hline
\end{tabular}




\begin{tabular}{|c|c|c|c|}
\hline $\mathrm{C}$ & 11.76516720 & 0.95521764 & 1.28797685 \\
\hline $\mathrm{C}$ & 12.98330438 & 1.84860989 & 1.15342627 \\
\hline $\mathrm{C}$ & 15.06499593 & 2.29777830 & 2.17500755 \\
\hline $\mathrm{C}$ & 14.95370004 & 3.73367872 & 2.66068636 \\
\hline $\mathrm{C}$ & 14.67412733 & 5.08831408 & 4.53589019 \\
\hline $\mathrm{C}$ & 14.31839348 & 5.04742463 & 6.00497725 \\
\hline $\mathrm{C}$ & 12.52042261 & 4.32860489 & 7.40630511 \\
\hline $\mathrm{C}$ & 11.23888888 & 3.53407143 & 7.26894317 \\
\hline $\mathrm{C}$ & 9.06799978 & 3.78877437 & 6.23172695 \\
\hline $\mathrm{C}$ & 5.17101618 & 3.10219078 & 3.53219966 \\
\hline $\mathrm{C}$ & 4.24386778 & 3.74780242 & 2.72773059 \\
\hline $\mathrm{C}$ & 2.90362471 & 3.78187677 & 3.08950799 \\
\hline $\mathrm{C}$ & 2.48128249 & 3.17923068 & 4.26723814 \\
\hline $\mathrm{C}$ & 3.40929059 & 2.55173994 & 5.08555843 \\
\hline $\mathrm{C}$ & 4.74786137 & 2.51786877 & 4.71556048 \\
\hline $\mathrm{C}$ & 8.57194390 & 9.48653982 & 2.02820453 \\
\hline $\mathrm{C}$ & 9.79367543 & 8.84181623 & 1.75255017 \\
\hline $\mathrm{C}$ & 10.68301059 & 8.52342014 & 2.79581491 \\
\hline $\mathrm{C}$ & 10.31305657 & 8.77545051 & 4.13077131 \\
\hline $\mathrm{C}$ & 9.07602291 & 9.38456067 & 4.40221272 \\
\hline $\mathrm{C}$ & 8.20083648 & 9.72919060 & 3.36010919 \\
\hline $\mathrm{C}$ & 8.65124634 & 9.71754868 & 5.80985729 \\
\hline $\mathrm{C}$ & 10.17257755 & 8.52943343 & 0.32335488 \\
\hline $\mathrm{C}$ & 6.88023439 & 10.37682221 & 3.70555922 \\
\hline $\mathrm{C}$ & 7.69129011 & 9.95799984 & 0.89248085 \\
\hline $\mathrm{C}$ & 12.04243946 & 7.97106463 & 2.42194625 \\
\hline $\mathrm{C}$ & 11.20238215 & 8.38065099 & 5.29279299 \\
\hline $\mathrm{N}$ & 10.64468069 & 5.56313394 & 4.00872616 \\
\hline $\mathrm{N}$ & 11.85230586 & 3.67420516 & 3.59361458 \\
\hline
\end{tabular}




$\begin{array}{llll}\mathrm{N} & 7.65891041 & 6.39864614 & 1.49027111 \\ \mathrm{O} & 9.66335187 & 2.11256771 & 4.63759512 \\ \mathrm{O} & 11.26531141 & 1.07542645 & 2.59887675 \\ \mathrm{O} & 13.86308892 & 1.56673046 & 2.21486127 \\ \mathrm{O} & 14.74959680 & 3.76507694 & 4.05630982 \\ \mathrm{O} & 12.99591357 & 4.56996299 & 6.10009968 \\ \mathrm{O} & 10.37500042 & 4.30321035 & 6.46794694 \\ \mathrm{O} & 6.15274676 & 5.19901839 & 5.64777526 \\ \mathrm{O} & 7.80977725 & 6.42156317 & 4.37037297 \\ \mathrm{O} & 5.59421584 & 6.14532531 & 3.49096758 \\ \mathrm{O} & 5.96048278 & 7.58534014 & 5.39288318 \\ \mathrm{~F} & 4.62001508 & 4.35002399 & 1.60519393 \\ \mathrm{~F} & 2.03425954 & 4.39366036 & 2.29050143 \\ \mathrm{~F} & 3.03941674 & 1.97611977 & 6.22325804 \\ \mathrm{~F} & 5.61006891 & 1.90560830 & 5.52539809 \\ \mathrm{~S} & 10.02181346 & 4.24437390 & 1.75944851 \\ \mathrm{Cl} & 6.37647602 & 6.34266564 & 4.73644965 \\ \mathrm{Br} & 0.61893886 & 3.18654586 & 4.72076206 \\ \mathrm{Br} & 7.02573255 & 3.10756423 & 3.05052986\end{array}$

\section{Crystal B:}

Cell parameters:
$\mathrm{a}=14.45283 \AA$
$\mathrm{b}=10.87395 \AA \quad \mathrm{c}=8.52816 \AA$
$\alpha=111.98854$
$\beta=101.84608$
$\gamma=56.79641$

Coordinates (Angstroms)

$\begin{array}{llll}\text { Atoms } & \mathrm{X} & \mathrm{Y} & \mathrm{Z}\end{array}$




\begin{tabular}{|c|c|c|c|}
\hline $\mathrm{H}$ & 11.92448183 & 2.80972461 & 5.39083363 \\
\hline $\mathrm{H}$ & 11.41461904 & 4.11041733 & 4.32768384 \\
\hline $\mathrm{H}$ & 12.67865588 & 0.90075461 & 4.50284184 \\
\hline $\mathrm{H}$ & 11.84850849 & 0.29701601 & 3.08792891 \\
\hline $\mathrm{H}$ & 9.36412658 & -0.85932575 & 6.11975981 \\
\hline $\mathrm{H}$ & 7.97230381 & 0.14695652 & 5.65092153 \\
\hline $\mathrm{H}$ & 7.85274033 & -0.60364561 & 3.46091661 \\
\hline $\mathrm{H}$ & 15.06155853 & 7.29693645 & 3.86745716 \\
\hline $\mathrm{H}$ & 14.92681433 & 7.69143360 & 1.45411986 \\
\hline $\mathrm{H}$ & 9.29315636 & 0.33797929 & 1.67882030 \\
\hline $\mathrm{H}$ & 16.82838009 & 8.59734310 & 0.07381151 \\
\hline $\mathrm{H}$ & 17.52248639 & 7.02155275 & 0.55200473 \\
\hline $\mathrm{H}$ & 17.52302451 & 5.94018844 & 7.77333047 \\
\hline $\mathrm{H}$ & 12.71715102 & 0.70919242 & 1.04484321 \\
\hline $\mathrm{H}$ & 6.87340798 & 8.62075896 & 0.83610073 \\
\hline $\mathrm{H}$ & 7.03892852 & 7.85704019 & 2.44354514 \\
\hline $\mathrm{H}$ & 14.40047108 & 1.57646772 & 2.16290484 \\
\hline $\mathrm{H}$ & 1.69545290 & 1.24245562 & 2.26637855 \\
\hline $\mathrm{H}$ & 1.83607402 & 2.20182544 & 4.52125914 \\
\hline $\mathrm{H}$ & 14.57608255 & 2.65251615 & 4.28660276 \\
\hline $\mathrm{H}$ & 15.20616868 & 2.41349384 & 6.71532866 \\
\hline $\mathrm{H}$ & 1.09381395 & 0.67361557 & 6.40676589 \\
\hline $\mathrm{H}$ & 13.71659154 & -0.12984511 & 7.67819951 \\
\hline $\mathrm{H}$ & 14.87603115 & 4.15877699 & 0.24605153 \\
\hline $\mathrm{H}$ & 12.94227810 & 2.64618218 & 0.14324999 \\
\hline $\mathrm{H}$ & 11.66726957 & -0.57534719 & 6.41251487 \\
\hline $\mathrm{H}$ & 9.15514303 & 1.21502327 & 7.46243489 \\
\hline $\mathrm{H}$ & 9.26088659 & 2.20892289 & 5.98599919 \\
\hline $\mathrm{H}$ & 9.73033413 & 4.42093694 & 2.67756098 \\
\hline $\mathrm{H}$ & 9.16969777 & 5.14344505 & 4.14752809 \\
\hline $\mathrm{H}$ & 9.75679148 & 6.11018208 & 2.90467330 \\
\hline $\mathrm{H}$ & 8.27960509 & 5.33504903 & 2.78445086 \\
\hline $\mathrm{H}$ & 5.55127699 & 6.21624343 & 6.43251372 \\
\hline $\mathrm{H}$ & 13.70440274 & 6.42884455 & 6.07143517 \\
\hline $\mathrm{H}$ & 12.30602293 & 5.42503083 & 6.40891681 \\
\hline $\mathrm{H}$ & 13.53043583 & 4.84401667 & 5.29249210 \\
\hline $\mathrm{H}$ & 14.11148021 & 4.72601789 & 3.51941069 \\
\hline $\mathrm{H}$ & 13.09036188 & 4.34175050 & 2.13385155 \\
\hline $\mathrm{H}$ & 14.29659507 & 5.63152701 & 2.02925493 \\
\hline $\mathrm{H}$ & 11.27790697 & 6.81985453 & 0.03960232 \\
\hline $\mathrm{H}$ & 12.95973123 & 7.24996950 & 0.29195637 \\
\hline $\mathrm{H}$ & 12.43594430 & 5.58502725 & 0.52740353 \\
\hline $\mathrm{H}$ & 10.63388027 & 8.59619985 & 0.43465282 \\
\hline $\mathrm{H}$ & 3.30446974 & -0.27976138 & 1.50690450 \\
\hline $\mathrm{H}$ & 4.68704289 & 0.80120322 & 1.60387007 \\
\hline $\mathrm{H}$ & 3.72690746 & 0.97847691 & 3.51897576 \\
\hline $\mathrm{H}$ & 2.67798426 & -0.29404159 & 4.16594539 \\
\hline $\mathrm{H}$ & 3.81433959 & 0.52901190 & 5.21916297 \\
\hline $\mathrm{H}$ & 5.94366903 & -1.23978520 & 6.89053411 \\
\hline $\mathrm{H}$ & 4.27972025 & -0.75491155 & 6.51704031 \\
\hline $\mathrm{H}$ & 10.60272945 & 6.65828668 & 6.74435823 \\
\hline
\end{tabular}




\begin{tabular}{|c|c|c|c|}
\hline $\mathrm{C}$ & 11.60808435 & 2.26952493 & 3.46078218 \\
\hline $\mathrm{C}$ & 9.07501129 & 0.09645000 & 5.64733929 \\
\hline $\mathrm{C}$ & 8.93879457 & -0.78182410 & 3.48980385 \\
\hline $\mathrm{C}$ & 9.48537045 & -0.66490832 & 2.08501291 \\
\hline $\mathrm{C}$ & 17.43563509 & 8.08064125 & 0.83100810 \\
\hline $\mathrm{C}$ & 18.80515504 & 8.73294807 & 0.85656615 \\
\hline $\mathrm{C}$ & 0.51597473 & -0.51122474 & 1.86141165 \\
\hline $\mathrm{C}$ & 0.69658195 & 0.84743351 & 2.51964200 \\
\hline $\mathrm{C}$ & 15.25030551 & 1.85093939 & 4.63619916 \\
\hline $\mathrm{C}$ & 14.96061783 & 1.54467009 & 6.08736941 \\
\hline $\mathrm{C}$ & 13.09877112 & 0.72447492 & 7.36166566 \\
\hline $\mathrm{C}$ & 11.67063677 & 0.27867997 & 7.10937487 \\
\hline $\mathrm{C}$ & 9.58711796 & 1.27604702 & 6.45448953 \\
\hline $\mathrm{C}$ & 6.02291332 & 3.27229814 & 4.08186954 \\
\hline $\mathrm{C}$ & 5.54332287 & 4.36883169 & 3.38141939 \\
\hline $\mathrm{C}$ & 4.34312760 & 4.96731348 & 3.74370783 \\
\hline $\mathrm{C}$ & 3.62725621 & 4.46035954 & 4.82065676 \\
\hline $\mathrm{C}$ & 4.11630615 & 3.37972758 & 5.54288502 \\
\hline $\mathrm{C}$ & 5.32005880 & 2.79434263 & 5.17797118 \\
\hline $\mathrm{C}$ & 10.99140630 & 7.96749585 & 2.50023623 \\
\hline $\mathrm{C}$ & 11.86945686 & 6.93232993 & 2.13446836 \\
\hline $\mathrm{C}$ & 12.52617107 & 6.17700613 & 3.13392073 \\
\hline $\mathrm{C}$ & 12.24944238 & 6.42968811 & 4.48879757 \\
\hline $\mathrm{C}$ & 11.30077831 & 7.41289842 & 4.85734900 \\
\hline $\mathrm{C}$ & 4.73074440 & -0.90574922 & 3.86567000 \\
\hline $\mathrm{C}$ & 12.98859396 & 5.73233521 & 5.61188066 \\
\hline $\mathrm{C}$ & 13.55939784 & 5.16254829 & 2.69112287 \\
\hline $\mathrm{C}$ & 12.14244970 & 6.62481218 & 0.67758076 \\
\hline $\mathrm{C}$ & 4.39859916 & -0.24535419 & 1.45302858 \\
\hline $\mathrm{C}$ & 3.68920322 & 0.13243076 & 4.21230097 \\
\hline $\mathrm{C}$ & 5.04071892 & -1.50644693 & 6.32804363 \\
\hline $\mathrm{N}$ & 11.72519177 & 3.16068644 & 4.45753800 \\
\hline $\mathrm{N}$ & 12.17142896 & 1.08882015 & 3.64104073 \\
\hline $\mathrm{N}$ & 9.24524066 & 5.24420851 & 3.11232450 \\
\hline $\mathrm{O}$ & 9.56759187 & 0.15884994 & 4.33134515 \\
\hline $\mathrm{O}$ & 16.83320523 & 8.18586160 & 2.10592116 \\
\hline $\mathrm{O}$ & 19.59027534 & 8.12837135 & 1.84924944 \\
\hline $\mathrm{O}$ & 0.57716032 & 0.67298288 & 3.90811913 \\
\hline $\mathrm{O}$ & 13.57447858 & 1.26142714 & 6.15059236 \\
\hline $\mathrm{O}$ & 10.99698997 & 1.37798461 & 6.53794065 \\
\hline $\mathrm{O}$ & 6.26058416 & 5.55962919 & 6.35499345 \\
\hline $\mathrm{O}$ & 8.62276917 & 5.32293822 & 5.85053839 \\
\hline $\mathrm{O}$ & 7.20037836 & 6.58898700 & 4.31016013 \\
\hline $\mathrm{O}$ & 1.76878767 & -1.55775726 & 6.53508593 \\
\hline $\mathrm{F}$ & 6.21132034 & 4.85035412 & 2.33535047 \\
\hline $\mathrm{F}$ & 18.33227202 & 5.98453477 & 3.04385077 \\
\hline $\mathrm{F}$ & 3.42359876 & 2.86707854 & 6.55751248 \\
\hline $\mathrm{F}$ & 5.76310067 & 1.75025653 & 5.87108288 \\
\hline S & 10.83172630 & 2.68300394 & 1.97695267 \\
\hline $\mathrm{S}$ & 7.55575718 & 6.34290239 & 5.71601862 \\
\hline $\mathrm{Br}$ & 16.38270315 & 5.22765532 & 5.26617490 \\
\hline
\end{tabular}




\section{Crystal C:}

Cell parameters:

$$
\begin{array}{lll}
\mathrm{a}=15.513828 \AA & \mathrm{b}=11.3904259 \AA & \mathrm{c}=8.4080956 \AA \\
\alpha=91.622135 & \beta=106.452642 & \gamma=97.878855
\end{array}
$$

Coordinates (Angstroms)

$\begin{array}{llll}\text { Atoms } & \mathrm{X} & \mathrm{Y} & \mathrm{Z}\end{array}$

$\begin{array}{cccc}\mathrm{H} & 8.42055920 & 6.64039844 & 4.94956428 \\ \mathrm{H} & 8.00400336 & 7.16294766 & 3.32542564 \\ \mathrm{H} & 9.14253000 & 4.55620134 & 5.29062089 \\ \mathrm{H} & 8.60065826 & 3.27690217 & 4.22350124 \\ \mathrm{H} & 6.43253765 & 3.41710952 & 7.39533693 \\ \mathrm{H} & 4.76715814 & 3.77034428 & 6.89422957 \\ \mathrm{H} & 4.76195989 & 2.06684870 & 5.13226286 \\ \mathrm{H} & 6.29097823 & 1.60518123 & 5.92478412 \\ \mathrm{H} & 5.99066743 & 0.79128579 & 3.55015333 \\ \mathrm{H} & 6.13227303 & 2.49125918 & 3.00729193 \\ \mathrm{H} & 7.77735629 & 1.39097451 & 1.82135963 \\ \mathrm{H} & 8.44024417 & 0.05812166 & 2.80651467 \\ \mathrm{H} & 10.22591536 & 1.18340056 & 1.60808948 \\ \mathrm{H} & 9.64111838 & 2.78108419 & 2.14586047 \\ \mathrm{H} & 12.27676959 & 1.92972189 & 2.48387504 \\ \mathrm{H} & 12.46612077 & 1.88395278 & 4.25058756 \\ \mathrm{H} & 10.98501721 & 4.17236494 & 2.88428882 \\ \mathrm{H} & 12.75676822 & 4.13827245 & 3.05795310 \\ \mathrm{H} & 12.69956188 & 5.99923934 & 4.60732373 \\ \mathrm{H} & 10.95747558 & 6.03835289 & 4.20935892 \\ \mathrm{H} & 11.51159291 & 7.13879695 & 6.37665276 \\ \mathrm{H} & 12.05128109 & 5.57057756 & 7.02787202 \\ \mathrm{H} & 12.50796465 & 6.58621515 & 0.57370126 \\ \mathrm{H} & 9.43396936 & 7.35000474 & 7.65275278 \\ \mathrm{H} & 10.01780578 & 6.65607840 & 0.79325636 \\ \mathrm{H} & 10.58340905 & 5.14732584 & 0.05555431 \\ \mathrm{H} & 5.58106039 & 5.73368984 & 7.83093599 \\ \mathrm{H} & 5.45023991 & 6.01324104 & 6.09097225 \\ \mathrm{H} & 5.28157928 & 5.56056841 & 2.66176182 \\ \mathrm{H} & 5.27855600 & 4.94080444 & 4.16288333 \\ \mathrm{H} & 5.75761545 & 6.47282760 & 3.89861219 \\ \mathrm{H} & 3.73156334 & 6.26779390 & 3.91096708 \\ \mathrm{H} & 0.93103052 & 6.38748045 & 6.51280007 \\ \mathrm{H} & 4.79135500 & 9.89512752 & 5.25069398\end{array}$




\begin{tabular}{|c|c|c|c|}
\hline $\mathrm{H}$ & 4.17136686 & 8.57717276 & 4.26230375 \\
\hline $\mathrm{H}$ & 5.23363233 & 8.23550228 & 5.62369291 \\
\hline $\mathrm{H}$ & 6.58757040 & 10.10716489 & 6.42009111 \\
\hline $\mathrm{H}$ & 7.56118943 & 8.64296489 & 6.56753827 \\
\hline $\mathrm{H}$ & 8.33911503 & 10.23215294 & 6.39851128 \\
\hline $\mathrm{H}$ & 11.57925368 & -0.32754796 & 4.83029254 \\
\hline $\mathrm{H}$ & 10.04372543 & 9.30134909 & 5.47046383 \\
\hline $\mathrm{H}$ & 10.82840739 & 9.65636923 & 3.93989252 \\
\hline $\mathrm{H}$ & 10.38394485 & 10.68024900 & 2.09944246 \\
\hline $\mathrm{H}$ & 10.58786246 & 8.99778860 & 1.60108432 \\
\hline $\mathrm{H}$ & 9.62527604 & 10.10778687 & 0.62034615 \\
\hline $\mathrm{H}$ & 4.88845427 & 9.33085325 & 7.86319666 \\
\hline $\mathrm{H}$ & 6.00436915 & 7.96598772 & 7.95980410 \\
\hline $\mathrm{H}$ & 4.27233619 & 7.68424082 & 7.98737038 \\
\hline $\mathrm{H}$ & 4.73506921 & 9.17927153 & 1.06013919 \\
\hline $\mathrm{H}$ & 5.22590469 & 7.49754940 & 1.23079536 \\
\hline $\mathrm{H}$ & 4.17632614 & 8.22775569 & 2.43726863 \\
\hline $\mathrm{C}$ & 8.30991741 & 5.16169248 & 3.54337546 \\
\hline $\mathrm{C}$ & 5.82462782 & 3.98381159 & 6.67497539 \\
\hline $\mathrm{C}$ & 5.85026004 & 2.22220949 & 5.12681199 \\
\hline $\mathrm{C}$ & 6.41074838 & 1.77978225 & 3.79421925 \\
\hline $\mathrm{C}$ & 8.39307456 & 1.14301518 & 2.69791345 \\
\hline $\mathrm{C}$ & 9.76420011 & 1.73382471 & 2.44733745 \\
\hline $\mathrm{C}$ & 11.82678607 & 2.24552687 & 3.43932588 \\
\hline $\mathrm{C}$ & 11.81244516 & 3.76583666 & 3.48531524 \\
\hline $\mathrm{C}$ & 11.70833771 & 5.61100228 & 4.89261005 \\
\hline $\mathrm{C}$ & 11.37872237 & 6.04914907 & 6.30238797 \\
\hline $\mathrm{C}$ & 9.49558461 & 6.25311304 & 7.74725275 \\
\hline $\mathrm{C}$ & 8.11570007 & 5.66144301 & 7.94077164 \\
\hline $\mathrm{C}$ & 6.01225289 & 5.47776364 & 6.85774806 \\
\hline $\mathrm{C}$ & 2.53258762 & 4.66285556 & 1.70855539 \\
\hline $\mathrm{C}$ & 1.29804404 & 5.29491897 & 1.67021975 \\
\hline $\mathrm{C}$ & 0.36685059 & 5.08546678 & 2.67918928 \\
\hline $\mathrm{C}$ & 0.65565851 & 4.23274916 & 3.73676648 \\
\hline $\mathrm{C}$ & 1.89589906 & 3.61122754 & 3.78910266 \\
\hline $\mathrm{C}$ & 2.82027624 & 3.82264871 & 2.77382704 \\
\hline $\mathrm{C}$ & 7.45594334 & 9.06624191 & 1.79942786 \\
\hline $\mathrm{C}$ & 8.63453330 & 9.47776327 & 2.44022390 \\
\hline $\mathrm{C}$ & 8.66482368 & 9.60211137 & 3.83977378 \\
\hline $\mathrm{C}$ & 7.48428638 & 9.43983532 & 4.57142194 \\
\hline $\mathrm{C}$ & 6.28198486 & 9.07277859 & 3.93109040 \\
\hline $\mathrm{C}$ & 6.27317795 & 8.84969765 & 2.54271297 \\
\hline $\mathrm{C}$ & 2.36078121 & 6.26828624 & 5.31383702 \\
\hline $\mathrm{C}$ & 5.04937527 & 8.93131012 & 4.79528764 \\
\hline $\mathrm{C}$ & 7.49978306 & 9.62067929 & 6.06780263 \\
\hline $\mathrm{C}$ & 9.96167143 & 9.89607892 & 4.55639141 \\
\hline $\mathrm{C}$ & 9.87117854 & 9.82868218 & 1.64417703 \\
\hline $\mathrm{C}$ & 7.43795444 & 8.92485357 & 0.29591297 \\
\hline $\mathrm{C}$ & 5.03166568 & 8.41861329 & 1.79111924 \\
\hline $\mathrm{N}$ & 8.32328401 & 6.44366847 & 3.95840023 \\
\hline $\mathrm{N}$ & 8.80704595 & 4.26255560 & 4.37837674 \\
\hline
\end{tabular}




$\begin{array}{lccc}\mathrm{N} & 5.04724051 & 5.78822324 & 3.63076147 \\ \mathrm{O} & 6.14346190 & 3.58533193 & 5.35362655 \\ \mathrm{O} & 7.81979487 & 1.69618737 & 3.87118599 \\ \mathrm{O} & 10.56761776 & 1.62978509 & 3.60455286 \\ \mathrm{O} & 11.69294141 & 4.20490675 & 4.82146981 \\ \mathrm{O} & 10.03598050 & 5.70105439 & 6.56868390 \\ \mathrm{O} & 7.35619860 & 5.94446161 & 6.78390311 \\ \mathrm{O} & 2.72776883 & 6.66418101 & 4.13446860 \\ \mathrm{O} & 2.99277155 & 5.53228649 & 6.06500169 \\ \mathrm{O} & 1.14798888 & 6.74866285 & 5.63965631 \\ \mathrm{~F} & 0.98503771 & 6.11036317 & 0.66538071 \\ \mathrm{~F} & -0.81190980 & 5.69841613 & 2.59954240 \\ \mathrm{~F} & 2.21360364 & 2.80121643 & 4.78916539 \\ \mathrm{~F} & 3.99845894 & 3.21046870 & 2.85490926 \\ \mathrm{~S} & 7.73358846 & 4.75556676 & 1.98052314 \\ \mathrm{Br} & -0.64820781 & 3.90376536 & 5.09727194 \\ \mathrm{Br} & 3.82906139 & 4.97750632 & 0.32641765\end{array}$

